\title{
33. PHYSICAL AND GEOTECHNICAL PROPERTIES OF SEDIMENTS FROM THE NORTHWEST PACIFIC: DEEP SEA DRILLING PROJECT LEG 86 ${ }^{1}$
}

\author{
P. J. Schultheiss, Institute of Oceanographic Sciences, Wormley²
}

\begin{abstract}
A wide variety of sediment types (red clays, carbonate oozes, and siliceous clays) were cored using the hydraulic piston corer on Leg 86 of the Deep Sea Drilling Project in the Northwest Pacific. Physical property data for all these sediments are presented in the form of tables and graphs. Measurements include bulk density, water content, gamma ray attenuation porosity evaluation (GRAPE), shear strength, compressional and shear wave velocities, consolidation, and permeability. Sample quality for the most part was considered good; however, some systematic downcore disturbance was found in the red clays. A significant difference between shipboard and shore-based gravimetric measurements was found and was correlated with sea state. The siliceous clays exhibited little progressive consolidation downhole, whereas the red clays were found to be normally consolidated below $20 \mathrm{~m}$. Vane shear strengths ranged from zero to $1700 \mathrm{~g} / \mathrm{cm}^{2}$ in red clay at a sub-bottom depth of $170 \mathrm{~m}$. Shear wave velocity correlated with vane shear strength and ranged up to $160 \mathrm{~m} / \mathrm{s}$ in the red clay.
\end{abstract}

\section{INTRODUCTION}

Leg 86 of the Deep Sea Drilling Project (DSDP) was primarily aimed at studying the oceanic paleoenvironment in the Western Pacific. The sites cored using the hydraulic piston corer (HPC) contained a wide variety of sediment types including thick sequences of red clays (Sites 576 and 578), calcareous oozes (Site 577), and siliceous clays (Sites 579 and 580). It was, therefore, a particularly good opportunity to examine and compare the physical properties of these different sediment types during a single cruise. In addition, at Site 576 , a complete hole (Hole 576A) was recovered and stored unsplit for geotechnical studies. The large amount of data produced by these studies is presented by the Geotechnical Consortium (this volume).

Data concerning physical and geotechnical properties of deep-sea sediments could substantially aid our understanding of depositional and post-depositional processes if "relatively undisturbed" samples are available. Prior to the introduction of the HPC, the rotary coring process was used to obtain sediment samples in the upper $200 \mathrm{~m}$ of unlithified sediments. The sample disturbance in these cores (Demars and Nacci, 1978) made the assessment and interpretation of some of the physical property data problematic. However, use of physical property data has greatly aided our understanding of sediment deposition and consolidation to great depths below the seafloor (e.g., Bennett and Keller, 1973; Lee, 1973; Rocker, 1974). DSDP Leg 68 (Prell, W. L., Gardner, J. V., et al., 1982) was the first cruise devoted to using the then newly developed HPC, which recovered sediment sections of $\sim 4 \mathrm{~m}$ length. Before this, the HPC had been successfully used only once (Leg 64, Site 480; Curray et al., 1979; Schrader et al., 1980).

\footnotetext{
${ }^{1}$ Heath, G. R., Burckle, L. H., et al., Init. Repts. DSDP, 86: Washington (U.S. Govt. Printing Office).

2 Address: Institute of Oceanographic Sciences, Wormley, Godalming, Surrey GU8 SUB, United Kingdom.
}

Mayer (1982) presented the first detailed physical property measurements made on HPC samples from depths of more than $200 \mathrm{~m}$ and provided an account of the problems of disturbance and the extent to which the HPC overcomes these problems. The observations that Mayer made with regard to the quality of HPC samples are equally applicable to the samples recovered on Leg 86 using the increased coring length $(9.5 \mathrm{~m})$ facility of the variable length hydraulic piston corer (VLHPC) and will not be repeated here in any detail.

The purpose of this chapter is to present all of the shipboard physical and geotechnical measurements made on Leg 86 as well as some of the shore-based data. Measurements made on board and on shore included bulk density, water content, shear strength, consolidation, permeability, and both compressional and shear wave velocities. After the techniques used have been described, the data are presented and discussed for each site before a comparison of the different sediment types is made.

\section{TECHNIQUES}

Apart from Site 581, all the measurements were made on cores obtained by the HPC. Each core, after having been sectioned at $1.5-\mathrm{m}$ intervals, was allowed to lie unsplit in the rack for at least $4 \mathrm{hr}$. in order to bring the temperature to equilibrium. This was nominally $21^{\circ} \mathrm{C}$ in the shipboard laboratory and all measurements were carried out close to this temperature. The only set of data corrected for temperature differences was the compressional wave velocity, which was corrected to $20^{\circ} \mathrm{C}$. The following measurements were carried out at varying intervals throughout the recovered cores.

\section{GRAPE}

All cores were passed through the Gamma Ray Attenuation and Porosity Evaluator (GRAPE) to assess the saturated bulk density (see Introduction and Explanatory Notes, this volume). The calibration and calculation procedures were explained by Boyce (1976). Samples used for the 2-min. GRAPE count were subsequently used for shipboard gravimetric analyses.

\section{Gravimetric Analysis}

Two adjacent samples were normally taken using thin stainless steel sampling cylinders (see Introduction and Explanatory Notes, this volume). One sample was subjected to the 2-min. GRAPE before being weighed wet, dried to constant weight at $105^{\circ} \mathrm{C}$, and then weighed dry. 
The second sample was sealed in a plastic vial which was then returned for a shore-based check on the data.

Water content (WC) was calculated from the wet and dry weights. $\mathrm{WC}=\mathrm{Wt}$. of salt water/Wt. of solids, using a salt correction of $35 \%$.

$$
\mathrm{WC}(\%)=\frac{103.5(W-D)}{D-0.036(W-D)},
$$

where $W=$ wet weight of sediment and $D=$ dry weight of sediment. Estimates of porosity, void ratio, and bulk density were obtained by assuming that the density of seawater is $1.024 \mathrm{~g} / \mathrm{cm}^{3}$ and the solid density is $2.7 \mathrm{~g} / \mathrm{cm}^{3}$.

\section{Compressional Wave Velocity}

The velocity of compressional waves was measured at around $400 \mathrm{kHz}$ using the Hamilton frame velocimeter. This equipment and the techniques for using and calibrating it were described by Boyce (1973, 1976). Most measurements were obtained by measuring across a split core section and through half of the liner (TTL). It was found that for HPC cores, where the sediments completely fill the liner, a resolution of better than $\pm 2 \%$ is possible; most measurements were probably accurate to within $\pm 1 \%$. Temperature was recorded at the time of each measurement, and the velocities were corrected to $20^{\circ} \mathrm{C}$ using tables of seawater velocity versus temperature.

\section{Shear Wave Velocity}

The general paucity of shear wave data to date has not been caused by a lack of interest but by a lack of suitable techniques for propagating and receiving shear waves in low velocity materials such as unconsolidated sediments. Although resonant column tests, as used by Hardin and Richart (1963), provide most of the shear wave data available, the technique is not suitable as a logging tool for split cores.

Over the last few years shear wave measurements in sediments have progressed significantly with the introduction of piezoelectric bender crystals being used as the transducers (Shirley and Hampton, 1978; Schultheiss, 1981, 1983). To transmit and receive shear waves in sediments, piezoelectric transducers have been constructed using thin bender elements, which are composite sheets consisting of two piezoelectric transverse expander plates bonded together face-to-face so that an applied voltage across the plates causes it to bend, as shown in Figure 1. This bending motion causes a shear stress that propagates as a shear wave and is detected in a reciprocal manner to develop in the surrounding sediment. The relatively large displacements that can be obtained for small applied voltages, coupled with the low resonant frequencies, make bender elements suitable as shear wave transducers for sediments under low effective stresses. For logging cores, a pair of transducers were built that were stiff enough to be pushed into the split core as shown in Figure 2. A perspex frame was used to push the transducers into the sediment. This enabled the elements to be inserted with the correct orientation and at a known separation. The frame was removed before readings were taken in order to decouple mechanically the transmitter and receiver. They were inserted both parallel and normal to the core axis to look for any anisotropic effects. The rising

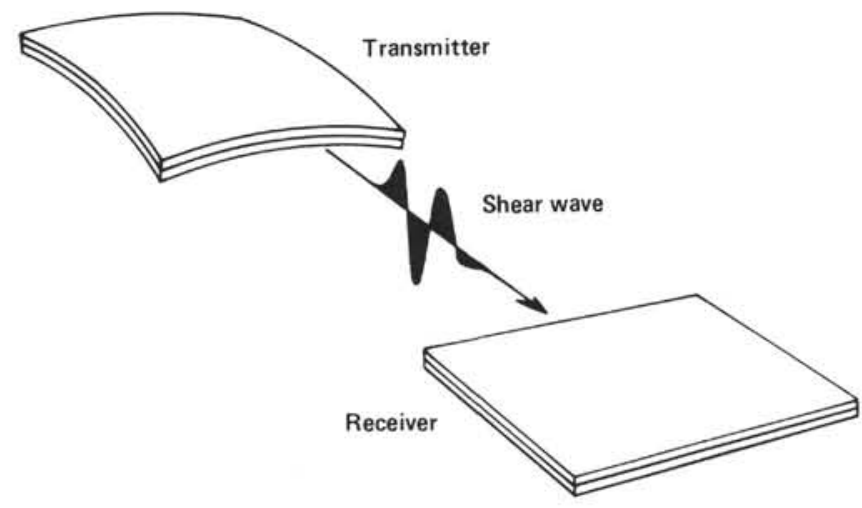

Figure 1. Schematic diagram of piezoelectric bender elements used to transmit and receive shear waves in unconsolidated sediments. edge of a 10-V square wave was used to drive the transmitter, which had a resonant frequency around $5 \mathrm{kHz}$. After passing through a highpass filter, the received signal was displayed on a delay time base oscilloscope which was used to measure the transit time. The accuracy of the velocity measurements depended primarily on the signal quality and was estimated at around $\pm 5 \%$.

\section{Shear Strength Measurements}

Two vane devices were routinely used to measure shear strength: the motorized Wykeham Farrance "Laboratory Vane Apparatus" and the Torvane hand-held vane. The theory, operation, and calculation procedures as used by DSDP for the motorized vane were well documented by Boyce (1977). Briefly, the motorized laboratory vane equipment has a motor that rotates $\left(89^{\circ} /\right.$ minute) one end of a calibrated, coiled spring about its axis, with the other end of the spring being connected to a shaft that incorporates a cruciform vane. A mechanical pointer system is used to indicate the amount of rotation of the vane and, in addition, the amount of rotational stress applied to the spring. At some maximum torque the vane begins to rotate more rapidly as the sediment shears around a cylinder described by the rotating vane. The shearing force is related to the maximum torque applied to this cylindrical shearing surface. It is assumed that, at fast rates of strain in clayey sediment with low permeabilities, the calculated shear strength is an undrained value. It should be emphasized here that it is most imperative to clamp rigidly the split core section to the vane apparatus to prevent any relative motions that would invalidate the results.

The Torvane hand-held vane operates on the same principles as the motorized vane except that the degree of control is much more limited and a full stress-strain graph cannot be produced. Only the maximum torque required to produce the failure is recorded. A great deal of care must be taken when using this device; the incorporation of well-engineered ball joints in the operator's wrist would be advantageous.

\section{RESULTS}

The data are presented in the form of a table and a series of graphs for each of the six sites (Sites 576-581). The columns in the tables are defined as follows:

Core-Section is self-explanatory; it refers to the core number followed by the section number (see Introduction and Explanatory Notes, this volume). Interval (cm) refers to the range of depths, within a given section, at which all the measurements were taken. Although each measurement was taken at a precisely known depth, they were all generally taken within $30 \mathrm{~cm}$ of one another in each section. Consequently, for ease of presentation and to allow parameters to be easily plotted against each other, most of the data within each section have been assigned an average sub-bottom depth (Av. sbd [m]) in meters below the seafloor. This value has been taken at the average of the depth intervals. In some cases, when data have been obtained from a part of the section outside a $50-\mathrm{cm}$ range or in an obviously different lithology, they have been assigned a unique interval and sub-bottom depth. The sub-bottom depth is the adjusted value which takes into account discrepancies that arose from the driller's log; in particular, for Site 576 (see Site 576 chapter, this volume).

Shear strength is separated into two columns; the first is for the hand-held Torvane and the second is for the Wykeham Farrance motorized vane device (W.F.). These are given in $\mathrm{g} / \mathrm{cm}^{2}$ for ease of comparison with other DSDP data. However, the preferred SI units are kilopascals $(\mathrm{kPa})$, where $10 \mathrm{~g} / \mathrm{cm}^{2}=0.918 \mathrm{kPa}$.

$\mathrm{P}$-wave velocity is the primary or compressional wave velocity measured at $400 \mathrm{kHz}$ and corrected to $20^{\circ} \mathrm{C}$ and given in $\mathrm{m} / \mathrm{s}$. 


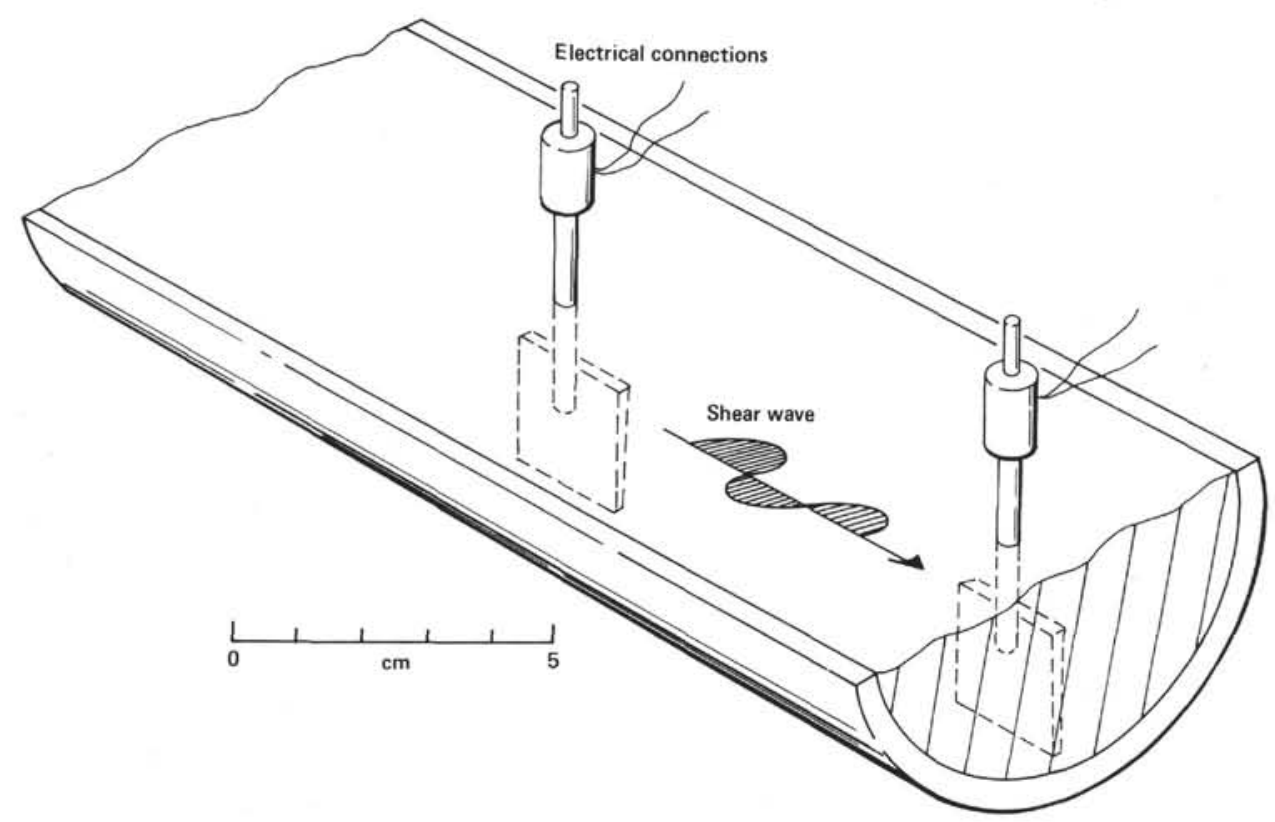

Figure 2. The shear wave transducers inserted into the split core section with the handling frame removed.

S-wave velocity is the secondary or shear wave velocity also given in $\mathrm{m} / \mathrm{s}$.

GRAPE bulk density is given in $\mathrm{g} / \mathrm{cm}^{3}$ and refers to the calculated density from the 2-min. GRAPE count.

Bulk Density, Water content, Porosity, and Void ratio have two sets of values; the first is from those measured on board ship (Shipboard) and the second is from those measured at the shore-based laboratory (Laboratory). Where more than one hole was drilled at a site, only the first hole has both sets of data; the other holes have just the shore-based laboratory data.

Most of the graphs have been plotted for each site with the data merged from all the holes at that site. The exception is Site 576, where Hole 576A was dedicated for geotechnical studies (Geotechnical Consortium, this volume). Bulk density data are plotted using the shorebased laboratory data unless stated. For each site, graphs of bulk density, water content, GRAPE bulk density, compressional wave velocity, shear wave velocity, and vane shear strength versus depth have been plotted (Figs. 3-8).

\section{Site 576}

This site consists of about $55 \mathrm{~m}$ of pelagic clay overlying $20 \mathrm{~m}$ of interbedded carbonate turbidites and pelagic clay before terminating in chert at $76 \mathrm{~m}$ sub-bottom. The thick pelagic clay is considered to be a "type" northwestern Pacific pelagic (red) clay section with continuous deposition throughout the past 65 m.y. Data for Holes 576 and 576B are presented in Table 1. A large amount of physical property and geotechnical data is presented by the Geotechnical Consortium (this volume) for Hole 576A.

The graphical representation of the data for this site is shown in Figure 3. The carbonate data points that lie between 55 and $72 \mathrm{~m}$ are indicated. The water content profile for this site exhibits high-frequency fluctuations which, although not yet understood, may reflect subtle changes in lithology and deposition. Water content over- all increases from around $150 \%$ near the surface to over $200 \%$ between 11 and $20 \mathrm{~m}$ before it gradually decreases to around $110 \%$ at $20 \mathrm{~m}$. It is suspected that the variations reflect changes in mineralogy similar to that found in LL44-GPC3 where the high water content is caused by increased amounts of authigenic components (probably smectite).

The compressional wave velocity profile (Fig. 3 ) shows no obvious trend through the brown clay section and is always less than the water velocity because of the high water content. Peaks in the profile correspond to ash layers and, toward the bottom of the core, the carbonate layers. The high velocity carbonates (coupled with their higher bulk densities) provide a large impedance contrast which explains the good acoustic reflector at the clay/ooze boundary (see Site 576 chapter, this volume).

The shear wave velocity profile for the brown clay shows an increase from around $10 \mathrm{~m} / \mathrm{s}$ at the surface to around $120 \mathrm{~m} / \mathrm{s}$ at a depth of $70 \mathrm{~m}$ (Fig. 3). Shear wave velocities in the carbonates around $60 \mathrm{~m}$ have only about half the value $(40 \mathrm{~m} / \mathrm{s})$ of the velocities in the adjacent clays $(80 \mathrm{~m} / \mathrm{s})$. A similar profile is shown for shear strength, which increases from less than $50 \mathrm{~g} / \mathrm{cm}^{2}$ at the surface to over $1000 \mathrm{~g} / \mathrm{cm}^{2}$ at $70 \mathrm{~m}$. Once again, the carbonates have much lower values than the adjacent clays. It is thought that the large differences in shear strength and shear wave velocity between the brown clay and the adjacent carbonates is probably caused by the stress disturbance that occurs during the coring operation rather than implying that the in situ values vary to a similar degree. The shear strength of a water-saturated sediment may be expressed by the Mohr-Coulomb relationship:

$$
s=\bar{c}+\bar{\delta} \tan \bar{\phi}
$$

where $\bar{c}=$ cohesion, $\bar{\phi}=$ effective angle of internal friction, and $\bar{\delta}=$ effective normal stress. To a large extent, the shear vane is measuring the cohesion only as 
Table 1. Physical property data for Holes 576, 576B, 577, 577A, 578, 579, 579A, 580, and 581.

\begin{tabular}{|c|c|c|c|c|c|c|c|c|c|c|c|c|c|}
\hline \multirow{3}{*}{$\begin{array}{l}\text { Core-Section } \\
\text { (interval or } \\
\text { level in } \mathrm{cm} \text { ) }\end{array}$} & \multirow{3}{*}{$\begin{array}{l}\text { Av. } \\
\text { sbd } \\
\text { (m) }\end{array}$} & \multicolumn{8}{|c|}{ Shipboard } & \multicolumn{4}{|c|}{ Laboratory } \\
\hline & & Shear strength & P-wave & S-wave & $\begin{array}{c}\text { GRAPE } \\
\text { bulk }\end{array}$ & Bulk & Water & & & & Water & & \\
\hline & & $\begin{array}{c}\text { Torvane W.F. } \\
\left(\mathrm{g} / \mathrm{cm}^{2}\right)\end{array}$ & $\begin{array}{l}\text { velocity } \\
(\mathrm{m} / \mathrm{s})\end{array}$ & $\begin{array}{l}\text { velocity } \\
(\mathrm{m} / \mathrm{s})\end{array}$ & $\begin{array}{l}\text { density } \\
\left(\mathrm{g} / \mathrm{cm}^{3}\right)\end{array}$ & $\begin{array}{l}\text { density } \\
\left(\mathrm{g} / \mathrm{cm}^{3}\right)\end{array}$ & $\begin{array}{c}\text { content } \\
(\%)\end{array}$ & Porosity & $\begin{array}{l}\text { Void } \\
\text { ratio }\end{array}$ & $\begin{array}{l}\text { density } \\
\left(\mathrm{g} / \mathrm{cm}^{3}\right)\end{array}$ & $\begin{array}{c}\text { content } \\
(\%)\end{array}$ & Porosity & $\begin{array}{l}\text { Void } \\
\text { ratio }\end{array}$ \\
\hline
\end{tabular}

Hole 576

\begin{tabular}{|c|c|c|c|c|c|c|c|c|c|c|c|c|c|c|}
\hline $1-1,58-84$ & 5.21 & 34 & 14 & - & - & 1.56 & - & - & - & - & 1.28 & 169 & 0.82 & 4.69 \\
\hline $1-2,64-78$ & 6.71 & 26 & - & - & - & 1.41 & 1.28 & 170 & 0.82 & 4.70 & 1.26 & 181 & 0.83 & 5.04 \\
\hline $2-1,21-79$ & 7.70 & 56 & 56 & 1439 & 4 & 1.46 & 1.31 & 151 & 0.81 & 4.18 & 1.31 & 151 & 0.81 & 4.20 \\
\hline $2-1,63$ & 7.83 & - & 32 & - & - & - & - & - & - & - & - & - & - & - \\
\hline $2-1,119$ & 8.39 & - & 54 & - & - & - & - & - & - & - & - & - & - & - \\
\hline $2-1,131$ & 8.51 & - & 39 & - & - & - & - & - & - & - & - & - & - & - \\
\hline $2-2,42-69$ & 9.25 & 46 & 54 & 1444 & 6 & 1.41 & 1.27 & 177 & 0.83 & 4.90 & 1.27 & 176 & 0.83 & 4.88 \\
\hline $2-2,91$ & 9.61 & - & - & 1443 & - & - & - & - & - & - & - & - & - & - \\
\hline $2-3,60-79$ & 10.89 & 74 & 82 & 1452 & 10 & 1.47 & 1.30 & 157 & 0.81 & 4.34 & 1.39 & 113 & 0.76 & 3.15 \\
\hline $2-4,12$ & 11.82 & - & 67 & - & - & - & - & - & - & - & - & - & - & - \\
\hline $2-4,42$ & 12.12 & - & 131 & - & - & - & - & - & - & - & - & - & - & - \\
\hline $2-4,90-109$ & 12.70 & 94 & 81 & 1445 & 12 & 1.39 & 1.27 & 173 & 0.83 & 4.79 & 1.27 & 176 & 0.83 & 4.87 \\
\hline $2-5,42-90$ & 13.86 & 88 & 91 & 1441 & 21 & 1.29 & 1.26 & 221 & 0.86 & 6.13 & 1.21 & 234 & 0.87 & 6.50 \\
\hline $2-6,12-69$ & 15.11 & 42 & 51 & 1440 & 12 & 1.33 & 1.22 & 216 & 0.86 & 5.98 & 1.22 & 218 & 0.86 & 6.05 \\
\hline $2-6,52$ & 15.22 & - & 37 & - & - & - & - & - & - & - & - & - & - & - \\
\hline $2-6,121$ & 15.91 & - & 11 & - & - & - & - & - & - & - & - & - & - & - \\
\hline $3-1,80-99$ & 10.50 & 96 & 60 & 1437 & 10 & 1.46 & 1.32 & 145 & 0.80 & 4.02 & 1.29 & 160 & 0.82 & 4.45 \\
\hline $3-2,60-79$ & 11.79 & 80 & 59 & 1435 & 13 & 1.34 & 1.22 & 214 & 0.86 & 5.92 & 1.23 & 212 & 0.85 & 5.89 \\
\hline $3-3,70-90$ & 13.40 & 104 & 67 & 1447 & 17 & 1.33 & 1.25 & 195 & 0.84 & 5.39 & 1.24 & 199 & 0.85 & 5.52 \\
\hline $3-4,40-80$ & 14.70 & 120 & 49 & 1424 & 16 & 1.38 & 1.27 & 175 & 0.83 & 4.85 & 1.26 & 179 & 0.83 & 4.98 \\
\hline $4-1,89-109$ & 20.08 & 92 & 68 & 1464 & 16 & 1.34 & 1.23 & 217 & 0.85 & 5.74 & 1.25 & 192 & 0.84 & 5.32 \\
\hline $4-2,32-80$ & 21.16 & 108 & 90 & 1445 & 17 & 1.36 & 1.28 & 166 & 0.82 & 4.60 & 1.27 & 172 & 0.83 & 4.77 \\
\hline $4-3,52-79$ & 22.76 & 128 & 111 & 1439 & 20 & 1.37 & 1.31 & 152 & 0.81 & 4.19 & 1.41 & 108 & 0.75 & 2.99 \\
\hline $4-4,40-80$ & 24.20 & 168 & 168 & 1479 & - & 1.45 & 1.35 & 131 & 0.78 & 3.62 & 1.33 & 137 & 0.79 & 3.80 \\
\hline $4-4,62-69$ & 24.26 & - & 137 & 1439 & - & - & - & - & - & - & - & - & - & - \\
\hline $4-5,52-79$ & 25.76 & 162 & 191 & 1433 & 26 & 1.55 & 1.33 & 138 & 0.79 & 3.83 & 1.33 & 140 & 0.80 & 3.89 \\
\hline $4-5,112$ & 26.22 & 190 & - & - & - & - & - & - & - & - & - & - & - & - \\
\hline $4-6,62-89$ & 27.36 & 280 & 227 & 1438 & 45 & 1.54 & 1.36 & 128 & 0.78 & 3.54 & 1.35 & 131 & 0.78 & 3.64 \\
\hline $5-1,52-99$ & 28.95 & 170 & 173 & 1425 & 44 & 1.50 & 1.36 & 125 & 0.77 & 3.45 & 1.33 & 137 & 0.79 & 3.80 \\
\hline $5-2,50-90$ & 30.40 & 95 & - & - & 34 & - & - & - & - & - & - & - & - & - \\
\hline $5-2,80$ & 30.50 & 120 & - & - & - & - & - & - & - & - & - & - & - & - \\
\hline $5-2,110$ & 30.80 & 110 & - & - & - & - & - & - & - & - & - & - & - & - \\
\hline $5-3,60-88$ & 31.94 & 110 & - & - & 37 & - & - & - & - & - & - & - & - & - \\
\hline $5-3,120$ & 32.40 & 150 & - & - & - & - & - & - & - & - & - & - & - & - \\
\hline $5-4,40$ & 33.10 & 120 & - & - & - & - & - & - & - & - & - & - & - & - \\
\hline $5-4,105$ & 33.75 & 110 & - & - & - & - & - & - & - & - & - & - & - & - \\
\hline $5-5,10-83$ & 37.57 & 130 & 100 & 1454 & 33 & 1.48 & 1.33 & 137 & 0.79 & 3.80 & 1.34 & 136 & 0.79 & 3.79 \\
\hline $5-5,52-79$ & 37.76 & 270 & 241 & 1415 & 51 & - & - & - & - & - & 1.33 & 142 & 0.80 & 3.94 \\
\hline $5-5,88$ & 37.98 & 260 & - & - & - & - & - & - & - & - & - & - & - & - \\
\hline $5-6,50-79$ & 39.24 & 220 & 205 & 1440 & 45 & 1.50 & 1.33 & 138 & 0.79 & 3.83 & 1.30 & 155 & 0.81 & 4.30 \\
\hline $6-3,59-93$ & 38.16 & 230 & 214 & 1427 & 55 & 1.42 & 1.37 & 122 & 0.77 & 3.37 & 1.36 & 127 & 0.78 & 3.54 \\
\hline $6-3,99$ & 38.39 & 230 & - & - & - & - & - & - & - & - & - & - & - & - \\
\hline $6-4,50-89$ & 39.59 & 340 & 205 & 1421 & 63 & - & 1.45 & 93 & 0.72 & 2.59 & 1.35 & 131 & 0.78 & 3.63 \\
\hline $6-5,70-90$ & 41.20 & 280 & - & 1424 & 58 & - & - & - & - & - & 1.33 & 139 & 0.79 & 3.85 \\
\hline $6-5,83$ & 41.23 & - & - & - & - & - & - & - & - & - & 1133 & 139 & 0.80 & 3.87 \\
\hline $6-6,62-89$ & 42.66 & 310 & 291 & 1431 & 63 & 1.47 & 1.38 & 119 & 0.77 & 3.29 & 1.35 & 128 & 0.78 & 3.56 \\
\hline $7-1,61-90$ & 45.36 & 260 & 275 & 1426 & 52 & 1.53 & 1.39 & 116 & 0.76 & 3.20 & - & - & - & - \\
\hline $7-2,61-94$ & 47.88 & 320 & 287 & 1428 & 75 & 1.44 & 1.37 & 122 & 0.77 & 3.37 & 1.41 & 108 & 0.75 & 3.00 \\
\hline $7-2,69-102$ & 47.95 & - & - & - & 65 & - & - & - & - & - & 1.36 & 125 & 0.78 & 3.46 \\
\hline $7-3,79-102$ & 49.51 & 380 & 469 & - & - & 1.40 & 1.35 & 129 & 0.78 & 3.56 & 1.33 & 141 & 0.80 & 3.93 \\
\hline $7-4,60-101$ & 50.91 & 420 & 419 & 1431 & 68 & 1.39 & 1.36 & 124 & 0.77 & 3.44 & 1.27 & 179 & 0.83 & 4.96 \\
\hline $7-5,30-70$ & 52.10 & 390 & 320 & - & 65 & 1.42 & 1.39 & 114 & 0.76 & 3.15 & 1.38 & 120 & 0.77 & 3.32 \\
\hline $7-6,71-99$ & 53.95 & 420 & 420 & - & 67 & 1.48 & 1.41 & 107 & 0.75 & 2.96 & 1.39 & 115 & 0.76 & 3.20 \\
\hline $8-1,30-70$ & 54.60 & 360 & 250 & 1428 & 60 & 1.31 & 1.37 & 122 & 6.77 & 3.37 & 1.36 & 128 & 0.78 & 3.54 \\
\hline $8-1,135$ & 55.45 & - & - & 1451 & - & - & - & - & - & - & - & - & - & - \\
\hline $8-2,47-101$ & 56.34 & 400 & 114 & 1464 & 59 & 1.62 & 1.62 & 61 & 0.63 & 1.69 & 1.65 & 55 & 0.61 & 1.55 \\
\hline $8-2,110-132$ & 56.81 & 510 & 453 & 1429 & - & - & - & - & - & - & 1.36 & 128 & 0.78 & 3.55 \\
\hline $8-3,20-139$ & 57.89 & 330 & 272 & 1448 & 62 & 1.69 & 1.59 & 66 & 0.65 & 1.81 & 1.57 & 68 & 0.66 & 1.90 \\
\hline $8-5,45-84$ & 60.74 & 150 & 59 & 1474 & 30 & 1.84 & 1.67 & 54 & 0.60 & 1.49 & 1.61 & 62 & 0.63 & 1.74 \\
\hline $8-6,15-63$ & 61.99 & - & 66 & 1508 & 31 & - & - & - & - & - & 1.66 & 54 & 0.60 & 1.51 \\
\hline $8-6,25-85$ & 62.15 & - & 552 & 1426 & 34 & - & - & - & - & - & 1.51 & 80 & 0.69 & 2.24 \\
\hline $8-6,80-109$ & 62.55 & - & - & 1480 & 75 & - & - & - & - & - & - & - & - & - \\
\hline
\end{tabular}

Hole 576B

\begin{tabular}{|c|c|c|c|c|c|c|c|c|c|c|c|c|c|c|}
\hline $1-1,72-99$ & 0.85 & 56 & 27 & 1444 & 11 & - & - & - & - & - & 1.29 & 159 & 0.82 & 4.42 \\
\hline $1-2,61-79$ & 2.20 & 14 & 1 & 1470 & - & - & - & - & - & - & 1.17 & 278 & 0.88 & 7.71 \\
\hline $1-3,60-89$ & 3.75 & 56 & 68 & 1452 & 15 & - & - & - & - & - & 1.30 & 154 & 0.81 & 4.28 \\
\hline $1-4,41-69$ & 5.05 & 44 & 27 & 1449 & 12 & - & - & - & - & - & 1.29 & 159 & 0.81 & 4.41 \\
\hline $1-5,82-109$ & 6.95 & 102 & 55 & 1458 & 14 & - & - & - & - & - & 1.28 & 169 & 0.83 & 4.70 \\
\hline $2-1,48-80$ & 12.24 & 70 & 57 & 1461 & 9 & - & - & - & - & - & 1.18 & 262 & 0.88 & 7.27 \\
\hline $2-2,60-102$ & 13.91 & 68 & 59 & - & 7 & - & - & - & - & - & 1.24 & 197 & 0.84 & 5.46 \\
\hline
\end{tabular}


Table 1. (Continued).

\begin{tabular}{|c|c|c|c|c|c|c|c|c|c|c|c|c|c|c|}
\hline \multirow[b]{2}{*}{$\begin{array}{l}\text { Core-Section } \\
\text { (interval or } \\
\text { level in } \mathrm{cm} \text { ) }\end{array}$} & \multirow[b]{2}{*}{$\begin{array}{l}\text { Av. } \\
\text { sbd } \\
\text { (m) }\end{array}$} & \multicolumn{9}{|c|}{ Shipboard } & \multicolumn{4}{|c|}{ Laboratory } \\
\hline & & $\frac{\text { Shear stre }}{\text { Torvane }}$ & $\frac{\text { ngth }}{\text { W.F. }}$ & $\begin{array}{l}\text { P-wave } \\
\text { velocity } \\
(\mathrm{m} / \mathrm{s})\end{array}$ & $\begin{array}{l}\text { S-wave } \\
\text { velocity } \\
(\mathrm{m} / \mathrm{s})\end{array}$ & $\begin{array}{c}\text { GRAPE } \\
\text { bulk } \\
\text { density } \\
\left(\mathrm{g} / \mathrm{cm}^{3}\right)\end{array}$ & $\begin{array}{c}\text { Bulk } \\
\text { density } \\
\left(\mathrm{g} / \mathrm{cm}^{3}\right)\end{array}$ & $\begin{array}{c}\text { Water } \\
\text { content } \\
(\%)\end{array}$ & Porosity & $\begin{array}{l}\text { Void } \\
\text { ratio }\end{array}$ & $\begin{array}{c}\text { Bulk } \\
\text { density } \\
\left(\mathrm{g} / \mathrm{cm}^{3}\right)\end{array}$ & $\begin{array}{c}\text { Water } \\
\text { content } \\
(\%)\end{array}$ & Porosity & $\begin{array}{l}\text { Void } \\
\text { ratio }\end{array}$ \\
\hline Hole 576B (Cont.) & & & & & & & & & & & & & & \\
\hline $2-3,69-102$ & 15.46 & 58 & 43 & 1465 & 21 & - & - & - & - & - & 1.23 & 212 & 0.85 & 5.87 \\
\hline $2-4,41-96$ & 21.29 & 82 & 62 & 1457 & 15 & - & - & - & - & - & 1.28 & 167 & 0.82 & 4.63 \\
\hline $2-5,31-89$ & 22.70 & 115 & 142 & 1521 & 63 & - & - & - & - & - & 1.28 & 167 & 0.82 & 4.64 \\
\hline $2-5,79-80$ & 22.89 & - & - & 1471 & - & - & - & - & - & - & 1.30 & 158 & 0.81 & 4.37 \\
\hline $2-6,62-89$ & 24.36 & 125 & 146 & 1459 & 62 & - & - & - & - & - & 1.30 & 156 & 0.81 & 4.34 \\
\hline $3-1,42-69$ & 21.65 & 140 & 107 & 1451 & 41 & - & - & - & - & - & 1.31 & 152 & 0.81 & 4.23 \\
\hline $3-1,69-73$ & 21.81 & - & 109 & 1448 & - & - & - & - & - & - & - & - & - & - \\
\hline $3-2,51-81$ & 23.26 & 122 & - & - & 41 & - & - & - & - & - & 1.32 & 146 & 0.80 & 4.04 \\
\hline $3-3,12-43$ & 24.38 & 138 & 105 & 1451 & 33 & - & - & - & - & - & 1.36 & 127 & 0.78 & 3.52 \\
\hline $3-4,101-146$ & 26.84 & 142 & - & 1444 & 30 & - & - & - & - & - & 1.37 & 122 & 0.77 & 3.40 \\
\hline $3-4,128$ & 26.88 & - & - & 1446 & - & - & - & - & - & - & - & - & - & - \\
\hline $3-5,44$ & 27.54 & - & - & - & 35 & - & - & - & - & - & - & - & - & - \\
\hline $3-5,83$ & 27.93 & - & - & - & 14 & - & - & - & - & - & - & - & - & - \\
\hline $3-5,57-115$ & 27.96 & 126 & - & 1445 & 41 & - & - & - & - & - & - & - & - & - \\
\hline $3-6,14$ & 28.74 & 140 & - & - & - & - & - & - & - & - & - & - & - & - \\
\hline $3-6,36-79$ & 29.17 & 230 & 209 & 1449 & 47 & - & - & - & - & - & 1.38 & 119 & 0.77 & 3.32 \\
\hline $4-4,61-78$ & 38.30 & 240 & 198 & 1448 & 50 & - & - & - & - & - & 1.34 & 132 & 0.79 & 3.68 \\
\hline $4-5,41-59$ & 39.60 & 260 & 227 & 1453 & 50 & - & - & - & - & - & 1.29 & 162 & 0.82 & 4.50 \\
\hline $4-6,61-81$ & 41.31 & 250 & 232 & 1444 & 51 & - & - & - & - & - & 1.31 & 150 & 0.81 & 4.17 \\
\hline $5-2,70-113$ & 42.51 & 240 & 237 & 1516 & 49 & - & - & - & - & - & 1.33 & 139 & 0.79 & 3.85 \\
\hline $5-2,100$ & 42.60 & - & - & - & 51 & - & - & - & - & - & - & - & - & - \\
\hline $5-3,70-91$ & 43.91 & 370 & - & 1463 & 58 & - & - & - & - & - & 1.35 & 129 & 0.78 & 3.59 \\
\hline $5-4,61-82$ & 45.32 & 410 & 337 & 1435 & 68 & - & - & - & - & - & 1.42 & 103 & 0.74 & 2.86 \\
\hline $5-5,62-81$ & 46.82 & 340 & 318 & 1452 & 65 & - & - & - & - & - & 1.40 & 110 & 0.75 & 3.05 \\
\hline $5-6,57-80$ & 48.28 & 320 & 318 & 1456 & 65 & - & - & - & - & - & 1.38 & 119 & 0.77 & 3.30 \\
\hline $6-2,61-82$ & 51.82 & 440 & 409 & 1453 & 68 & - & - & - & - & - & 1.36 & 127 & 0.78 & 3.53 \\
\hline $6-3,62-81$ & 53.32 & 390 & - & 1455 & 68 & - & - & - & - & - & 1.40 & 111 & 0.76 & 3.09 \\
\hline $6-4,61-82$ & 54.82 & 550 & 519 & 1456 & 72 & - & - & - & - & - & 1.35 & 130 & 0.78 & 3.61 \\
\hline $6-5,34-76$ & 56.15 & - & 141 & 1504 & 38 & - & - & - & - & - & 1.68 & 52 & 0.59 & 1.45 \\
\hline $6-5,95$ & 56.55 & - & - & - & 72 & - & - & - & - & - & - & - & - & - \\
\hline $7-1,59-82$ & 59.78 & 190 & 55 & 1492 & 41 & - & - & - & - & - & 1.64 & 58 & 0.62 & 1.61 \\
\hline $7-5,106-124$ & 68.15 & 250 & - & 1505 & 40 & - & - & - & - & - & 1.68 & 51 & 0.59 & 1.44 \\
\hline $8-2,73-98$ & 69.81 & 900 & 707 & 1512 & 121 & - & - & - & - & - & 1.73 & 45 & 0.56 & 1.27 \\
\hline $8-2,89$ & 69.84 & - & - & 1474 & - & - & - & - & - & - & 1.37 & 122 & 0.77 & 3.38 \\
\hline $8-3,69-84$ & 71.22 & 1000 & 1060 & 1480 & 127 & - & - & - & - & - & 1.26 & 182 & 0.84 & 5.05 \\
\hline Hole 577 & & & & & & & & & & & & & & \\
\hline $1-1,20-66$ & 0.43 & 36 & - & 1506 & - & 1.72 & 1.48 & 88 & 0.71 & 2.44 & 1.47 & 90 & 0.72 & 2.51 \\
\hline $1-1,72$ & 0.72 & 94 & - & - & - & - & - & - & - & - & - & - & - & - \\
\hline $1-3,74-100$ & 3.87 & 121 & 184 & 1472 & 39 & 1.63 & 1.42 & 104 & 0.74 & 2.88 & 1.46 & 93 & 0.72 & 2.58 \\
\hline $1-4,12-26$ & 4.69 & 380 & 147 & 1470 & - & 1.66 & 1.51 & 79 & 0.69 & 2.21 & 1.50 & 82 & 0.70 & 2.28 \\
\hline $2-2,69-77$ & 9.04 & 210 & - & 1502 & 36 & - & - & - & - & - & 1.51 & 80 & 0.69 & 2.22 \\
\hline $2-4,54-74$ & 11.95 & 380 & 140 & 1520 & 35 & 1.53 & 1.46 & 92 & 0.72 & 2.55 & 1.55 & 72 & 0.67 & 2.00 \\
\hline $2-6,58-94$ & 15.07 & 330 & 191 & 1492 & 36 & 1.63 & 1.51 & 80 & 0.69 & 2.21 & 1.60 & 63 & 0.64 & 1.75 \\
\hline $3-2,78-84$ & 18.62 & 130 & - & 1477 & - & - & - & - & - & - & 1.47 & 89 & 0.71 & 2.48 \\
\hline $3-4,60-80$ & 21.51 & 480 & 169 & 1480 & 53 & 1.72 & 1.56 & 71 & 0.66 & 1.97 & 1.59 & 64 & 0.64 & 1.79 \\
\hline $3-5,60$ & 22.91 & - & - & 1477 & - & - & - & - & - & - & - & - & - & - \\
\hline $3-5,56-73$ & 22.95 & 420 & 206 & 1480 & 44 & 1.67 & 1.58 & 66 & 0.65 & 2.69 & 1.55 & 72 & 0.67 & 2.00 \\
\hline $4-3,79-100$ & 29.70 & 450 & 276 & 1476 & 53 & 1.69 & - & - & - & - & - & - & - & - \\
\hline $4-4,66-85$ & 31.07 & 340 & 213 & 1486 & 35 & 1.50 & 1.59 & 66 & 0.64 & 1.81 & 1.57 & 68 & 0.66 & 1.90 \\
\hline $4-6,49-68$ & 33.89 & 400 & 133 & 1467 & 34 & 1.67 & 1.54 & 74 & 0.67 & 2.16 & 1.54 & 74 & 0.67 & 2.07 \\
\hline $5-2,66-85$ & 37.57 & 400 & 280 & 1470 & 41 & 1.75 & 1.58 & 66 & 0.65 & 1.83 & 1.57 & 68 & 0.66 & 1.91 \\
\hline $5-4,68-82$ & 40.56 & 580 & 339 & 1467 & 64 & 1.66 & 1.57 & 69 & 0.66 & 1.91 & 1.58 & 66 & 0.65 & 1.84 \\
\hline $5-5,67-85$ & 42.07 & 550 & 317 & 1473 & 69 & 1.76 & 1.58 & 66 & 0.65 & 1.83 & 1.55 & 72 & 0.67 & 2.00 \\
\hline $6-1,69-85$ & 45.58 & 410 & - & 1472 & 44 & 1.65 & 1.55 & 72 & 0.67 & 1.99 & 1.56 & 70 & 0.66 & 1.95 \\
\hline $6-3,33-85$ & 48.40 & 410 & 294 & 1472 & 43 & 1.59 & 1.59 & 65 & 0.64 & 1.80 & 1.55 & 72 & 0.67 & 2.01 \\
\hline $6-5,66$ & 51.47 & - & - & 1479 & - & - & - & - & - & - & - & - & - & - \\
\hline $6-5,56-79$ & 51.49 & 560 & 427 & 1479 & 67 & 1.69 & 1.56 & 71 & 0.66 & 1.97 & 1.56 & 70 & 0.66 & 1.95 \\
\hline $6-6,69-87$ & 53.09 & 380 & - & 1484 & 43 & 1.67 & 1.60 & 63 & 0.64 & 1.75 & 1.59 & 65 & 0.65 & 1.82 \\
\hline $7-1,62-86$ & 55.05 & 300 & 258 & - & 57 & 1.72 & 1.55 & 73 & 0.67 & 2.01 & 1.53 & 77 & 0.68 & 2.14 \\
\hline $7-3,62-81$ & 58.03 & 320 & 302 & - & 55 & 1.69 & 1.58 & 68 & 0.65 & 1.87 & 1.57 & 68 & 0.65 & 1.89 \\
\hline $7-5,55-81$ & 60.99 & 260 & 250 & 1516 & - & 1.82 & 1.72 & 48 & 0.57 & 1.33 & 1.70 & 49 & 0.58 & 1.38 \\
\hline $8-1,68-87$ & 64.59 & 250 & 52 & 1492 & - & 1.83 & 1.66 & 55 & 0.60 & 1.51 & 1.66 & 55 & 0.61 & 1.54 \\
\hline $8-3,78-95$ & 67.68 & 180 & 29 & 1506 & - & 1.92 & 1.72 & 48 & 0.57 & 1.32 & 1.68 & 52 & 0.59 & 1.45 \\
\hline $8-5,62-98$ & 70.61 & 110 & 35 & 1605 & - & 1.82 & 1.72 & 48 & 0.57 & 1.32 & 1.67 & 53 & 0.60 & 1.48 \\
\hline $9-1,64-100$ & 74.13 & 96 & 27 & 1607 & - & 1.79 & 1.72 & 47 & 0.57 & 1.31 & 1.72 & 47 & 0.57 & 1.31 \\
\hline $9-3,66-98$ & 77.13 & 115 & 43 & 1533 & - & 1.81 & 1.71 & 49 & 0.57 & 1.34 & 1.68 & 51 & 0.59 & 1.44 \\
\hline $9-6,51-87$ & 81.50 & 88 & 18 & - & 58 & 1.93 & 1.66 & 56 & 0.61 & 1.54 & 1.66 & 54 & 0.60 & 1.52 \\
\hline $10-1,70$ & 83.51 & - & - & - & - & 1.78 & - & - & - & - & - & - & - & - \\
\hline $10-2,66-96$ & 85.12 & 112 & 31 & 1525 & - & - & 1.71 & 48 & 0.57 & 1.33 & 1.69 & 51 & 0.59 & 1.42 \\
\hline $10-4,55-88$ & 88.02 & 142 & 16 & - & 64 & 1.45 & 1.72 & 48 & 0.57 & 1.33 & 1.73 & 46 & 0.56 & 1.30 \\
\hline $10-6,52-100$ & 91.07 & 142 & 16 & 1510 & 61 & 1.78 & 1.72 & 48 & 0.57 & 1.33 & 1.69 & 51 & 0.59 & 1.42 \\
\hline
\end{tabular}


Table 1. (Continued).

\begin{tabular}{|c|c|c|c|c|c|c|c|c|c|c|c|c|c|c|}
\hline \multirow{3}{*}{$\begin{array}{l}\text { Core-Section } \\
\text { (interval or } \\
\text { level in } \mathrm{cm} \text { ) }\end{array}$} & \multirow{3}{*}{$\begin{array}{l}\text { Av. } \\
\text { sbd } \\
(\mathrm{m})\end{array}$} & \multicolumn{9}{|c|}{ Shipboard } & \multicolumn{4}{|c|}{ Laboratory } \\
\hline & & \multicolumn{2}{|c|}{ Shear strength } & & S-wave & $\begin{array}{c}\text { GRAPE } \\
\text { bulk }\end{array}$ & & & & & & Water & & \\
\hline & & $\begin{array}{l}\text { Torvane } \\
\qquad\left(\mathrm{g} / \mathrm{cm}^{2}\right)\end{array}$ & W.F. & $\begin{array}{l}\text { velocity } \\
(\mathrm{m} / \mathrm{s})\end{array}$ & $\begin{array}{c}\text { velocity } \\
(\mathrm{m} / \mathrm{s})\end{array}$ & $\begin{array}{l}\text { density } \\
\left(\mathrm{g} / \mathrm{cm}^{3}\right)\end{array}$ & $\begin{array}{l}\text { density } \\
\left(\mathrm{g} / \mathrm{cm}^{3}\right)\end{array}$ & $\begin{array}{c}\text { content } \\
(\%)\end{array}$ & Porosity & $\begin{array}{l}\text { Void } \\
\text { ratio }\end{array}$ & $\begin{array}{l}\text { density } \\
\left(\mathrm{g} / \mathrm{cm}^{3}\right)\end{array}$ & $\begin{array}{c}\text { content } \\
(\%)\end{array}$ & Porosity & $\begin{array}{l}\text { Void } \\
\text { ratio }\end{array}$ \\
\hline Hole 577 (Cont.) & & & & & & & & & & & & & & \\
\hline $11-1,71-101$ & 93.17 & 180 & - & 1529 & 39 & 1.79 & 1.72 & 47 & 0.56 & 1.30 & 1.72 & 48 & 0.57 & 1.33 \\
\hline $11-4,100-117$ & 97.90 & 200 & - & 1516 & - & - & 1.72 & 47 & 0.57 & 1.32 & 1.69 & 50 & 0.58 & 1.40 \\
\hline $11-5,110-122$ & 99.47 & 200 & - & 1502 & - & - & - & - & - & - & 1.67 & 53 & 0.60 & 1.48 \\
\hline Hole $577 \mathrm{~A}$ & & & & & & & & & & & & & & . \\
\hline $1-4,3-16$ & 4.60 & 420 & 125 & 1524 & 41 & - & - & - & - & - & 1.51 & 80 & 0.69 & 2.23 \\
\hline $1-6,122-136$ & 8.79 & 310 & 18 & 1471 & 59 & - & - & - & - & - & 1.39 & 113 & 0.76 & 3.14 \\
\hline $2-4,3-18$ & 14.00 & 435 & 118 & 1485 & 49 & - & - & - & - & - & 1.49 & 85 & 0.70 & 2.37 \\
\hline $3-2,5-19$ & 20.52 & 150 & 74 & 1494 & 30 & - & - & - & - & - & 1.51 & 80 & 0.69 & 2.23 \\
\hline $3-4,128-137$ & 24.72 & 355 & - & 1483 & 50 & - & - & - & - & - & 1.55 & 73 & 0.67 & 2.03 \\
\hline $4-4,3-28$ & 33.05 & 550 & 98 & 1491 & 43 & - & - & - & - & - & 1.57 & 69 & 0.66 & 1.91 \\
\hline $4-6,114-137$ & 37.16 & 290 & 74 & 1485 & 32 & - & - & - & - & - & 1.59 & 65 & 0.64 & 1.81 \\
\hline $5-2,122-137$ & 40.70 & 215 & 51 & 1474 & 28 & - & - & - & - & - & 1.58 & 66 & 0.65 & 1.84 \\
\hline $5-6,4-20$ & 45.52 & 580 & 297 & 1465 & 55 & - & - & - & - & - & 1.55 & 72 & 0.67 & 2.00 \\
\hline $6-3,124-137$ & 51.70 & 500 & 431 & 1468 & 65 & - & - & - & - & - & 1.53 & 77 & 0.68 & 2.13 \\
\hline $6-6,122-136$ & 56.19 & 350 & 261 & 1482 & 42 & - & - & - & - & - & 1.56 & 70 & 0.66 & 1.94 \\
\hline $7-2,109-133$ & 59.61 & 250 & 86 & 1464 & 41 & - & - & - & - & - & 1.54 & 73 & 0.67 & 2.04 \\
\hline $7-6,107-128$ & 65.57 & 124 & 41 & 1521 & 58 & - & - & - & - & - & 1.69 & 51 & 0.59 & 1.43 \\
\hline $8-1,118-135$ & 67.66 & 100 & 33 & 1537 & - & - & - & - & - & - & 1.69 & 50 & 0.58 & 1.40 \\
\hline $8-4,104-135$ & 72.10 & 92 & 29 & 1524 & 46 & - & - & - & - & - & 1.70 & 50 & 0.58 & 1.39 \\
\hline $9-2,120-135$ & 78.68 & 62 & 8 & 1607 & - & - & - & - & - & - & 1.69 & 50 & 0.58 & 1.40 \\
\hline $10-3,114-133$ & 89.64 & 68 & 3 & 1531 & - & - & - & - & - & - & 1.73 & 46 & 0.56 & 1.28 \\
\hline $10-6,2-6$ & 92.94 & 370 & - & 1513 & 36 & - & - & - & - & - & 1.67 & 54 & 0.60 & 1.50 \\
\hline $11-4,4-19$ & 99.52 & 104 & 14 & 1524 & 23 & - & - & - & - & - & 1.68 & 52 & 0.59 & 1.46 \\
\hline $11-6,128-136$ & 103.72 & 152 & 62 & 1504 & 36 & - & - & - & - & - & 1.65 & 56 & 0.61 & 1.57 \\
\hline $12-3,12-29$ & 107.60 & 114 & 103 & 1595 & 27 & - & - & - & - & - & 1.54 & 73 & 0.67 & 2.03 \\
\hline $12-5,120-142$ & 111.71 & 126 & 27 & 1522 & 46 & - & - & - & - & - & 1.72 & 47 & 0.57 & 1.31 \\
\hline $13-4,7-23$ & 118.55 & 116 & 16 & 1515 & 28 & - & - & - & - & - & 1.68 & 51 & 0.59 & 1.43 \\
\hline $13-6,114-130$ & 122.62 & 108 & 16 & 1507 & 43 & - & - & - & - & - & 1.67 & 53 & 0.60 & 1.49 \\
\hline Hole 578 & & & & & & & & & & & & & & \\
\hline $1-1,120-140$ & 1.30 & 28 & 21 & 1460 & - & 1.56 & 1.25 & 189 & 0.84 & 5.22 & 1.34 & 134 & 0.79 & 3.72 \\
\hline $1-3,112-138$ & 4.25 & 54 & 55 & 1461 & - & 1.59 & 1.31 & 151 & 0.81 & 4.19 & 1.30 & 158 & 0.81 & 4.39 \\
\hline $2-2,77-100$ & 7.19 & 60 & 37 & 1563 & - & 1.56 & 1.28 & 166 & 0.82 & 4.59 & 1.26 & 179 & 0.83 & 4.98 \\
\hline $2-2,93$ & 7.23 & - & - & 1485 & - & - & - & - & - & - & - & - & - & - \\
\hline $2-2,111$ & 7.41 & - & - & 1562 & - & - & - & - & - & - & - & - & - & - \\
\hline $2-3,50-71$ & 8.40 & 120 & 231 & 1474 & 28 & 1.44 & 1.29 & 164 & 0.82 & 4.55 & 1.25 & 188 & 0.84 & 5.21 \\
\hline $2-3,93$ & 8.73 & - & - & 1485 & - & - & - & - & - & - & - & - & - & - \\
\hline $2-4,73-97$ & 10.15 & 410 & 199 & 1468 & 51 & 1.49 & 1.31 & 149 & 0.80 & 4.12 & 1.30 & 159 & 0.81 & 4.41 \\
\hline $2-4,122$ & 10.52 & 110 & - & - & - & - & - & - & - & - & - & - & - & - \\
\hline $3-2,89-110$ & 16.80 & 165 & 156 & 1468 & 43 & 1.57 & 1.25 & 192 & 0.84 & 5.30 & 1.25 & 191 & 0.84 & 5.31 \\
\hline $3-3,70-71$ & 18.01 & - & - & 1490 & - & - & - & - & - & - & 1.30 & 157 & 0.81 & 4.37 \\
\hline $3-3,90$ & 18.20 & - & - & 1470 & - & - & - & - & - & - & - & - & - & - \\
\hline $3-3,110$ & 18.40 & - & - & 1524 & - & - & - & - & - & - & - & - & - & - \\
\hline $3-3,113$ & 18.43 & - & - & 1653 & - & - & - & - & - & - & - & - & - & - \\
\hline $3-3,134$ & 18.64 & - & - & 1505 & - & - & - & - & - & - & - & - & - & - \\
\hline $3-4,102-122$ & 19.92 & 235 & 277 & 1465 & 43 & 1.53 & 1.33 & 141 & 0.80 & 3.90 & 1.32 & 144 & 0.80 & 4.00 \\
\hline $3-5,63$ & 20.93 & - & - & 1770 & - & - & - & - & - & - & - & - & - & - \\
\hline $3-6,81-102$ & 22.71 & 190 & 154 & 1468 & 39 & 1.50 & 1.32 & 144 & 0.80 & 3.99 & 1.33 & 142 & 0.80 & 3.94 \\
\hline $3-6,113$ & 22.93 & - & - & 1653 & - & - & - & - & - & - & 1.77 & 42 & 0.54 & 1.18 \\
\hline $3-6,117$ & 22.97 & - & - & 1462 & - & - & - & - & - & - & - & - & - & - \\
\hline $4-2,49-70$ & 25.89 & 270 & 133 & - & 41 & 1.45 & 1.33 & 139 & 0.79 & 3.84 & 1.32 & 143 & 0.80 & 3.96 \\
\hline $4-3,20-141$ & 27.61 & 310 & 283 & 1642 & 73 & 1.59 & - & - & - & - & 1.39 & 112 & 0.76 & 3.11 \\
\hline $4-3,129$ & 28.09 & - & - & 1475 & - & - & - & - & - & - & - & - & - & - \\
\hline $4-5,60-82$ & 30.51 & 370 & 197 & 1460 & 58 & 1.49 & 1.34 & 137 & 0.79 & 3.78 & 1.33 & 140 & 0.80 & 3.88 \\
\hline $5-2,21-42$ & 35.11 & 210 & 131 & 1469 & 39 & 1.48 & 1.33 & 138 & 0.79 & 3.81 & 1.36 & 123 & 0.77 & 3.43 \\
\hline $5-3,58-82$ & 37.00 & 390 & 353 & 1478 & - & 1.36 & 1.34 & 135 & 0.80 & 3.72 & 1.34 & 133 & 0.79 & 3.70 \\
\hline $5-5,70-98$ & 40.14 & 285 & 320 & 1468 & 46 & - & - & - & - & - & 1.35 & 130 & 0.78 & 3.62 \\
\hline $5-5,74-104$ & 40.19 & - & 343 & - & - & - & - & - & - & - & 1.32 & 146 & 0.80 & 4.05 \\
\hline $5-6,77$ & 41.57 & - & - & - & 55 & - & - & - & - & - & - & - & - & - \\
\hline $6-2,61-94$ & 45.07 & 450 & 308 & 1468 & 91 & 1.40 & 1.32 & 145 & 0.80 & 4.02 & 1.32 & 146 & 0.80 & 4.05 \\
\hline $6-3,16$ & 45.96 & - & - & 1671 & - & - & - & - & - & - & - & - & - & - \\
\hline $6-3,53$ & 46.33 & - & - & 1630 & - & - & - & - & - & - & - & - & - & - \\
\hline $6-3,69$ & 46.49 & - & - & 1431 & - & - & - & - & - & - & - & - & - & - \\
\hline $6-3,116$ & 46.96 & - & - & 1569 & - & - & - & - & - & - & - & - & - & - \\
\hline $6-3,123$ & 47.03 & - & - & 1470 & - & - & - & - & - & - & - & - & - & - \\
\hline $6-3,129$ & 47.09 & - & - & 1554 & - & - & - & - & - & - & - & - & - & - \\
\hline $6-3,144$ & 47.24 & - & - & 1463 & - & - & - & - & - & - & - & - & - & - \\
\hline $6-4,72-100$ & 48.16 & 415 & 466 & 1473 & 38 & 1.35 & 1.26 & 181 & 0.83 & 5.00 & 1.27 & 179 & 0.83 & 4.97 \\
\hline $6-6,61-82$ & 51.01 & 315 & 279 & 1469 & 39 & 1.39 & 1.29 & 160 & 0.81 & 4.42 & 1.28 & 170 & 0.83 & 4.73 \\
\hline $7-2,81-97$ & 54.69 & 275 & 168 & - & - & 1.30 & 1.27 & 173 & 0.83 & 4.78 & 1.28 & 165 & 0.82 & 4.58 \\
\hline
\end{tabular}


Table 1. (Continued).

\begin{tabular}{|c|c|c|c|c|c|c|c|c|c|c|c|c|c|c|}
\hline \multirow[b]{2}{*}{$\begin{array}{l}\text { Core-Section } \\
\text { (interval or } \\
\text { level in } \mathrm{cm} \text { ) }\end{array}$} & \multirow[b]{2}{*}{$\begin{array}{l}\text { Av. } \\
\text { sbd } \\
\text { (m) }\end{array}$} & \multicolumn{9}{|c|}{ Shipboard } & \multicolumn{4}{|c|}{ Laboratory } \\
\hline & & $\begin{array}{l}\text { Shear strer } \\
\begin{array}{l}\text { Torvane } \\
\left(\mathrm{g} / \mathrm{cm}^{2}\right.\end{array}\end{array}$ & $\frac{\text { ength }^{\text {W.F. }}}{\text { 2) }}$ & $\begin{array}{l}\text { P-wave } \\
\text { velocity } \\
\text { (m/s) }\end{array}$ & $\begin{array}{c}\text { S-wave } \\
\text { velocity } \\
(\mathrm{m} / \mathrm{s})\end{array}$ & $\begin{array}{c}\text { GRAPE } \\
\text { bulk } \\
\text { density } \\
\left(\mathrm{g} / \mathrm{cm}^{3}\right)\end{array}$ & $\begin{array}{l}\text { Bulk } \\
\text { density } \\
\left(\mathrm{g} / \mathrm{cm}^{3}\right)\end{array}$ & $\begin{array}{c}\text { Water } \\
\text { content } \\
(\%)\end{array}$ & Porosity & $\begin{array}{l}\text { Void } \\
\text { ratio }\end{array}$ & $\begin{array}{l}\text { Bulk } \\
\text { density } \\
\left(\mathrm{g} / \mathrm{cm}^{3}\right)\end{array}$ & $\begin{array}{l}\text { Water } \\
\text { content } \\
(\%)\end{array}$ & Porosity & $\begin{array}{l}\text { Void } \\
\text { ratio }\end{array}$ \\
\hline Hole 578 (Cont.) & & & & & & & & & & & & & & \\
\hline $7-4,60-80$ & 57.50 & 260 & 421 & 1470 & - & 1.37 & 1.28 & 170 & 0.83 & 4.71 & 1.27 & 179 & 0.83 & 4.96 \\
\hline $7-6,69-101$ & 60.65 & 380 & 412 & 1468 & - & 1.43 & 1.32 & 144 & 0.80 & 3.00 & 1.29 & 161 & 0.82 & 4.46 \\
\hline $8-2,89-101$ & 64.25 & 345 & - & 1461 & - & 1.37 & 1.29 & 161 & 0.82 & 4.45 & 1.30 & 154 & 0.81 & 4.29 \\
\hline $8-4,111-122$ & 67.47 & 350 & 431 & 1486 & - & 1.73 & 1.43 & 100 & 0.73 & 2.76 & 1.38 & 117 & 0.76 & 3.24 \\
\hline $8-6,62-80$ & 70.01 & 305 & 242 & 1508 & 57 & 1.42 & 1.42 & 105 & 0.74 & 2.90 & 1.48 & 86 & 0.71 & 2.40 \\
\hline $9-2,110-133$ & 74.02 & 420 & 232 & 1463 & 49 & 1.41 & 1.34 & 134 & 0.79 & 3.71 & 1.33 & 140 & 0.80 & 3.89 \\
\hline $9-4,109-132$ & 77.01 & 400 & 261 & 1454 & 51 & 1.38 & 1.32 & 144 & 0.80 & 3.97 & 1.33 & 142 & 0.80 & 3.94 \\
\hline $9-6,69-582$ & 82.06 & 430 & 332 & 1470 & 53 & 1.46 & 1.35 & 123 & 0.77 & 3.40 & 1.35 & 129 & 0.78 & 3.59 \\
\hline $10-2,90-111$ & 83.31 & 460 & 283 & 1448 & 64 & 1.37 & 1.35 & 132 & 0.78 & 3.65 & 1.34 & 136 & 0.79 & 3.78 \\
\hline $10-4,99-122$ & 86.40 & 510 & 523 & 1464 & 64 & 1.36 & 1.32 & 144 & 0.80 & 3.99 & 1.31 & 152 & 0.81 & 4.23 \\
\hline $10-4,131$ & 86.61 & - & 449 & - & - & - & - & - & - & - & - & - & - & - \\
\hline $10-6,50-72$ & 88.91 & 520 & 375 & 1469 & 61 & 1.35 & 1.33 & 138 & 0.79 & 3.83 & 1.32 & 142 & 0.80 & 3.95 \\
\hline $11-2,110-130$ & 93.00 & 530 & 346 & 1462 & 64 & 1.29 & 1.33 & 138 & 0.79 & 3.82 & 1.32 & 143 & 0.80 & 3.96 \\
\hline $11-2,137$ & 93.17 & - & 324 & - & - & - & - & - & - & - & - & - & - & - \\
\hline $11-4,90-113$ & 95.81 & - & 431 & - & 64 & 1.46 & 1.33 & 137 & 0.79 & 3.79 & 1.33 & 142 & 0.80 & 3.94 \\
\hline $11-6,69-93$ & 98.61 & 640 & 441 & 1466 & 64 & 1.40 & 1.36 & 126 & 0.78 & 3.49 & 1.37 & 122 & 0.77 & 3.39 \\
\hline $12-2,27-113$ & 102.00 & 605 & 443 & 1463 & 65 & 1.34 & 1.34 & 134 & 0.79 & 3.71 & 1.64 & 57 & 0.61 & 1.58 \\
\hline $12-2,90$ & 102.20 & - & - & - & - & - & - & - & - & - & 1.33 & 138 & 0.79 & 3.84 \\
\hline $12-5,90-113$ & 106.81 & 720 & 632 & 1465 & 85 & 1.34 & 1.35 & 129 & 0.78 & 3.56 & 1.34 & 133 & 0.79 & 3.69 \\
\hline $13-2,77-112$ & 111.74 & 700 & 464 & 1459 & 64 & 1.40 & 1.31 & 148 & 0.80 & 4.09 & 1.31 & 149 & 0.81 & 4.15 \\
\hline $13-4,110-124$ & 114.97 & 550 & 449 & 1459 & 62 & 1.27 & 1.33 & 141 & 0.80 & 3.91 & 1.32 & 143 & 0.80 & 3.98 \\
\hline $13-6,73-110$ & 117.72 & 575 & 497 & 1480 & 65 & 1.50 & 1.38 & 117 & 0.76 & 3.24 & 1.38 & 116 & 0.76 & 3.24 \\
\hline $14-2,60-114$ & 121.17 & 775 & 479 & 1469 & 85 & 2.01 & 1.35 & 130 & 0.78 & 3.60 & 1.32 & 143 & 0.80 & 3.97 \\
\hline $14-4,80-114$ & 124.27 & 1025 & 1104 & 1467 & 98 & 1.98 & 1.38 & 117 & 0.77 & 3.25 & 1.38 & 117 & 0.76 & 3.24 \\
\hline $14-6,59-106$ & 127.13 & 850 & 1237 & 1471 & 91 & 2.10 & 1.47 & 90 & 0.71 & 2.49 & 1.40 & 110 & 0.75 & 3.05 \\
\hline $15-2,79-112$ & 130.75 & 888 & 1023 & 1469 & 128 & 1.93 & 1.45 & 94 & 0.72 & 2.60 & 1.43 & 99 & 0.73 & 2.77 \\
\hline $15-4,77-112$ & 133.75 & - & 1082 & 1479 & 121 & 2.04 & 1.48 & 88 & 0.71 & 2.44 & 1.47 & 90 & 0.71 & 2.50 \\
\hline $15-6,77-112$ & 136.75 & 900 & 1001 & 1482 & 128 & 2.00 & 1.47 & 89 & 0.71 & 2.47 & 1.47 & 89 & 0.71 & 2.49 \\
\hline $16-2,78-116$ & 140.27 & 625 & 982 & 1484 & 128 & - & 1.51 & 81 & 0.69 & 2.23 & 1.52 & 77 & 0.68 & 2.15 \\
\hline $16-3,95$ & 141.75 & - & - & - & - & 1.69 & - & - & - & - & - & - & - & - \\
\hline $16-4,90-112$ & 143.31 & 950 & 913 & 1477 & 128 & 1.65 & 1.47 & 91 & 0.72 & 2.51 & 1.44 & 96 & 0.73 & 2.67 \\
\hline $17-1,90-112$ & 148.31 & 1025 & 994 & 1474 & - & 1.48 & 1.49 & 86 & 0.70 & 2.37 & 1.47 & 91 & 0.72 & 2.53 \\
\hline $17-3,88-114$ & 151.31 & 975 & 1001 & 1468 & - & 1.45 & 1.47 & 89 & 0.71 & 2.47 & 1.47 & 88 & 0.71 & 2.46 \\
\hline $17-5,89-116$ & 154.33 & 1050 & 1215 & 1468 & - & 1.49 & 1.44 & 99 & 0.73 & 2.75 & 1.42 & 103 & 0.74 & 2.87 \\
\hline $18-3,90-113$ & 160.82 & 1500 & 1413 & 1510 & 134 & 1.79 & 1.64 & 58 & 0.62 & 1.61 & 1.61 & 61 & 0.63 & 1.70 \\
\hline $18-4,92-113$ & 162.33 & 1600 & 1539 & 1468 & - & - & - & - & - & - & 1.60 & 63 & 0.64 & 1.77 \\
\hline $19-1,90-111$ & 167.31 & 1650 & 1200 & 1491 & 21 & 1.71 & 1.60 & 64 & 0.64 & 1.78 & 1.57 & 69 & 0.66 & 1.91 \\
\hline $19-3,89-111$ & 170.30 & 1700 & 1325 & 1519 & 159. & 1.66 & 1.60 & 63 & 0.64 & 1.75 & 1.59 & 65 & 0.64 & 1.81 \\
\hline $20-1,24-41$ & 176.13 & 1700 & 1303 & 1527 & - & 2.17 & 1.59 & 66 & 0.65 & 1.82 & 1.56 & 70 & 0.66 & 1.94 \\
\hline $20-4,36$ & 180.66 & - & - & - & 142 & - & - & - & - & - & - & - & - & - \\
\hline Hole 579 & & & & & & & & & & & & & & \\
\hline $1-2,112-122$ & 2.67 & 24 & 23 & 1484 & - & - & 1.22 & 220 & 0.86 & 6.09 & 1.21 & 231 & 0.87 & 6.40 \\
\hline $1-5,114-123$ & 7.19 & 44 & 41 & 1485 & - & - & 1.26 & 186 & 0.84 & 5.08 & 1.27 & 1.75 & 0.83 & 4.85 \\
\hline $2-2,124-145$ & 11.25 & 96 & 86 & 1493 & 18 & - & 1.30 & 157 & 0.81 & 4.34 & 1.28 & 169 & 0.82 & 4.70 \\
\hline $2-5,85-88$ & 15.26 & - & - & 1475 & - & - & 1.28 & 167 & 0.82 & 4.62 & 1.30 & 159 & 0.81 & 4.40 \\
\hline Hole 579A & & & & & & & & & & & & & & \\
\hline $1-3,60-105$ & 17.83 & 210 & 265 & 1474 & 43 & 1.38 & 1.29 & 184 & 0.84 & 5.08 & 1.27 & 173 & 0.83 & 4.80 \\
\hline $1-5,84-116$ & 21.00 & 200 & 125 & 1472 & 41 & 1.41 & 1.31 & 150 & 0.81 & 4.14 & 1.31 & 153 & 0.81 & 4.24 \\
\hline $2-3,68-122$ & 27.45 & 250 & 96 & 1488 & 46 & 1.50 & 1.35 & 131 & 0.78 & 3.62 & 1.28 & 167 & 0.82 & 4.63 \\
\hline $2-5,64-96$ & 30.30 & 220 & 193 & 1477 & 40 & 1.45 & 1.32 & 145 & 0.80 & 4.00 & 1.31 & 148 & 0.80 & 4.11 \\
\hline $3-2,57-84$ & 35.20 & 160 & 107 & 1488 & 32 & - & 1.34 & 132 & 0.79 & 3.66 & 1.40 & 111 & 0.76 & 3.09 \\
\hline $3-5,42-78$ & 39.60 & 240 & 304 & 1499 & 44 & 1.45 & 1.40 & 111 & 0.77 & 3.07 & 1.38 & 118 & 0.77 & 3.28 \\
\hline $4-1,69-110$ & 43.39 & 160 & 176 & 1474 & 35 & 1.35 & 1.27 & 176 & 0.83 & 4.88 & - & - & - & - \\
\hline $4-2,69$ & 44.69 & - & - & - & - & - & - & - & - & - & 1.27 & 173 & 0.83 & 4.80 \\
\hline $4-3,77-109$ & 46.43 & 200 & 193 & 1480 & 40 & - & 1.27 & 175 & 0.73 & 4.83 & 1.27 & 174 & 0.83 & 4.83 \\
\hline $5-2,80-96$ & 54.38 & 80 & 47 & 1462 & - & 1.39 & 1.29 & 164 & 0.72 & 4.63 & 1.28 & 165 & 0.82 & 4.59 \\
\hline $5-5,8$ & 58.08 & - & - & 1606 & - & - & - & - & - & - & - & - & - & - \\
\hline $5-5,19$ & 58.19 & - & - & 1500 & - & - & - & - & - & - & - & - & - & - \\
\hline $5-5,69-88$ & 58.78 & 410 & 332 & 1484 & 46 & 1.42 & 1.29 & 162 & 0.82 & 4.49 & 1.26 & 183 & 0.84 & 5.08 \\
\hline $6-3,74-109$ & 65.41 & 460 & 328 & 1484 & 53 & 1.39 & 1.27 & 175 & 0.83 & 4.85 & 1.28 & 169 & 0.82 & 4.68 \\
\hline $6-5,14-57$ & 67.85 & 380 & 380 & 1491 & 46 & 1.41 & 1.30 & 157 & 0.81 & 4.33 & 1.28 & 166 & 0.82 & 4.61 \\
\hline $6-5,83-99$ & 68.41 & 100 & 107 & 1473 & - & - & 1.33 & 140 & 0.80 & 3.89 & 1.29 & 161 & 0.82 & 4.46 \\
\hline $7-2,99-117$ & 73.58 & 240 & 185 & 1484 & 40 & 1.40 & 1.24 & 197 & 0.84 & 5.46 & 1.25 & 190 & 0.84 & 5.26 \\
\hline $7-4,120-136$ & 76.78 & 380 & - & 1486 & - & 1.42 & 1.30 & 157 & 0.71 & 4.35 & 1.29 & 164 & 0.82 & 4.55 \\
\hline $8-1,98$ & 81.48 & - & - & 1478 & - & - & - & - & - & - & - & - & - & - \\
\hline $8-1,102-114$ & 81.58 & 470 & 250 & 1462 & - & 1.39 & 1.31 & 151 & 0.81 & 4.17 & 1.31 & 152 & 0.81 & 4.23 \\
\hline $8-5,121-137$ & 87.79 & 470 & 349 & 1488 & - & - & 1.28 & 166 & 0.72 & 4.60 & 1.28 & 172 & 0.83 & 4.76 \\
\hline $9-2,67-76$ & 92.22 & 425 & 293 & 1472 & - & 1.33 & 1.25 & 195 & 0.84 & 5.40 & 1.25 & 188 & 0.84 & 5.22 \\
\hline $9-5,48-73$ & 96.60 & 1000 & 807 & 1491 & - & 1.35 & 1.24 & 200 & 0.85 & 5.54 & 1.24 & 195 & 0.84 & 5.42 \\
\hline
\end{tabular}


Table 1. (Continued).

\begin{tabular}{|c|c|c|c|c|c|c|c|c|c|c|c|c|c|c|}
\hline \multirow{3}{*}{$\begin{array}{l}\text { Core-Section } \\
\text { (interval or } \\
\text { level in } \mathrm{cm} \text { ) }\end{array}$} & \multirow{3}{*}{$\begin{array}{l}\text { Av. } \\
\text { sbd } \\
\text { (m) }\end{array}$} & \multicolumn{9}{|c|}{ Shipboard } & \multicolumn{4}{|c|}{ Laboratory } \\
\hline & & \multicolumn{2}{|c|}{ Shear strength } & \multirow{2}{*}{$\begin{array}{l}\text { P-wave } \\
\text { velocity } \\
(\mathrm{m} / \mathrm{s})\end{array}$} & \multirow{2}{*}{$\begin{array}{l}\text { S-wave } \\
\text { velocity } \\
(\mathrm{m} / \mathrm{s})\end{array}$} & \multirow{2}{*}{$\begin{array}{l}\text { GRAPE } \\
\text { bulk } \\
\text { density } \\
\left(\mathrm{g} / \mathrm{cm}^{3}\right)\end{array}$} & \multirow{2}{*}{$\begin{array}{c}\text { Bulk } \\
\text { density } \\
\left(\mathrm{g} / \mathrm{cm}^{3}\right)\end{array}$} & \multirow{2}{*}{$\begin{array}{c}\text { Water } \\
\text { content } \\
(\%)\end{array}$} & \multirow[b]{2}{*}{ Porosity } & \multirow[b]{2}{*}{$\begin{array}{l}\text { Void } \\
\text { ratio }\end{array}$} & \multirow{2}{*}{$\begin{array}{c}\text { Bulk } \\
\text { density } \\
\left(\mathrm{g} / \mathrm{cm}^{3}\right)\end{array}$} & \multirow{2}{*}{$\begin{array}{c}\text { Water } \\
\text { content } \\
(\%)\end{array}$} & \multirow[b]{2}{*}{ Porosity } & \multirow[b]{2}{*}{$\begin{array}{l}\text { Void } \\
\text { ratio }\end{array}$} \\
\hline & & $\begin{array}{l}\text { Torvane } \\
\qquad\left(\mathrm{g} / \mathrm{cm}^{2}\right)\end{array}$ & W.F. & & & & & & & & & & & \\
\hline \multicolumn{15}{|l|}{ Hole 579A (Cont.) } \\
\hline $10-3,100-112$ & 103.56 & 750 & 604 & 1483 & - & 1.33 & 1.24 & 228 & 0.86 & 6.32 & 1.23 & 212 & 0.85 & 5.89 \\
\hline $10-5,57-67$ & 106.12 & 500 & 559 & 1495 & - & 1.39 & 1.26 & 181 & 0.83 & 5.01 & 1.24 & 199 & 0.85 & 5.53 \\
\hline $11-2,104-126$ & 111.65 & 575 & 633 & 1480 & - & - & 1.23 & 213 & 0.85 & 5.89 & 1.22 & 216 & 0.86 & 5.98 \\
\hline $11-4,73-100$ & 114.36 & 725 & 670 & 1474 & - & - & 1.34 & 199 & 0.85 & 5.49 & 1.23 & 212 & 0.85 & 5.88 \\
\hline $12-2,97-114$ & 121.06 & 650 & 456 & 1476 & - & 1.36 & 1.24 & 201 & 0.85 & 5.56 & 1.22 & 222 & 0.86 & 6.16 \\
\hline $12-4,79-111$ & 123.95 & 688 & 788 & 1480 & - & - & 1.22 & 214 & 0.86 & 5.93 & 1.23 & 206 & 0.85 & 5.73 \\
\hline $13-2,91-115$ & 130.53 & 750 & 434 & 1477 & - & 1.33 & 1.22 & 223 & 0.86 & 6.18 & 1.21 & 228 & 0.86 & 6.33 \\
\hline $13-4,79-114$ & 133.47 & 650 & 456 & 1481 & - & 1.32 & 1.21 & 228 & 0.86 & 6.30 & - & - & - & - \\
\hline $14-2,70-104$ & 139.87 & 525 & 339 & 1478 & - & 1.30 & 1.23 & 208 & 0.85 & 5.76 & - & - & - & - \\
\hline $14-4,66-107$ & 142.86 & 575 & 471 & 1485 & - & 1.37 & 1.25 & 194 & 0.84 & 5.38 & 1.23 & 205 & 0.85 & 5.68 \\
\hline $15-2,43-57$ & 149.00 & 625 & 552 & 1476 & - & 1.44 & 1.30 & 158 & 0.81 & 4.38 & 1.28 & 165 & 0.82 & 4.59 \\
\hline
\end{tabular}

Hole 580

$\begin{array}{lr}1-2,105-117 & 2.61 \\ 1-2,137 & 2.87 \\ 2-2,60-85 & 5.52 \\ 2-4,107-125 & 8.96 \\ 3-3,70-84 & 16.57 \\ 3-6,82-94 & 21.18 \\ 4-2,59-108 & 24.63 \\ 4-4,78-115 & 27.77 \\ 5-1,67 & 32.47 \\ 5-2,80-109 & 34.24 \\ 5-4,67-102 & 37.14 \\ 6-2,91-121 & 43.86 \\ 6-5,62-89 & 48.05 \\ 7-2,72-115 & 53.24 \\ 7-4,70-120 & 56.25 \\ 8-2,83-94 & 62.68 \\ 8-5,94-105 & 67.30 \\ 8-5,103 & 67.33 \\ 8-5,111 & 67.41 \\ 8-5,115 & 67.45 \\ 9-2,29-55 & 71.72 \\ 9-2,57 & 71.87 \\ 9-2,59 & 71.89 \\ 9-2,61 & 71.91 \\ 9-2,63 & 71.93 \\ 9-2,65 & 71.95 \\ 9-2,67 & 71.97 \\ 9-2,69 & 71.99 \\ 9-2,71 & 72.01 \\ 9-2,73 & 72.03 \\ 9-2,75 & 72.05 \\ 9-2,77 & 72.07 \\ 9-2,79 & 72.09 \\ 9-2,81 & 72.11 \\ 9-2,83 & 72.13 \\ 9-2,85 & 72.15 \\ 9-2,87 & 72.17 \\ 9-3,32 & 73.12 \\ 9-5,73-86 & 76.60 \\ 10-2,112-122 & 81.97 \\ 10-5,108-120 & 86.44 \\ 11-4,112-122 & 94.47 \\ 12-2,74-92 & 100.63 \\ 12-5,66-76 & 105.01 \\ 13-2,39-96 & 109.98 \\ 13-5,71-85 & 114.58 \\ 14-2,101-119 & 119.90 \\ 14-5,76-85 & 124.10 \\ 15-2,74-115 & 129.25 \\ 15-5,66-83 & 133.55 \\ 16-2,82-159 & 139.00 \\ 16-5,110-121 & 143.45 \\ 17-2,125-133 & 148.59 \\ 17-5,121-129 & 153.05 \\ 9 & \end{array}$

$\begin{array}{lll}72 & 41 & 1473 \\ - & - & 1646 \\ 48 & 34 & 1479 \\ 74 & 51 & 1479 \\ 92 & 50 & 1486 \\ 128 & 83 & 1470 \\ 102 & 68 & 1465 \\ 164 & 119 & 1488 \\ - & - & - \\ 240 & 121 & 1464 \\ 260 & 193 & 1486 \\ 250 & 152 & 1479 \\ 330 & 209 & 1472 \\ 380 & 277 & 1476 \\ 440 & 285 & 1467 \\ 400 & 258 & 1488 \\ 550 & 368 & 1489 \\ - & - & 1512 \\ - & - & 1586 \\ - & - & 1473 \\ 300 & 236 & 1486 \\ - & - & 1486 \\ - & - & 1543 \\ - & - & 1477 \\ - & - & 1476 \\ - & - & 1474 \\ - & - & 1493 \\ - & - & 1492 \\ - & - & 1489 \\ - & - & 1497 \\ - & - & 1507 \\ - & - & 1524 \\ - & - & 1559 \\ - & - & 1600 \\ - & - & 1603 \\ - & - & 1616 \\ - & - & - \\ 465 & 361 & 1484 \\ 650 & 471 & 1486 \\ 575 & 567 & 1491 \\ 520 & 353 & 1477 \\ 475 & 375 & 1508 \\ 550 & 545 & 1482 \\ 475 & 464 & 1479 \\ 520 & 449 & 1484 \\ 380 & 368 & 1485 \\ 450 & 795 & 1506 \\ 600 & 699 & 1488 \\ 680 & 596 & 1494 \\ 655 & 567 & 1498 \\ 715 & 714 & 1507 \\ 860 & 891 & 1490 \\ 570 & 589 & 1490 \\ & & \end{array}$

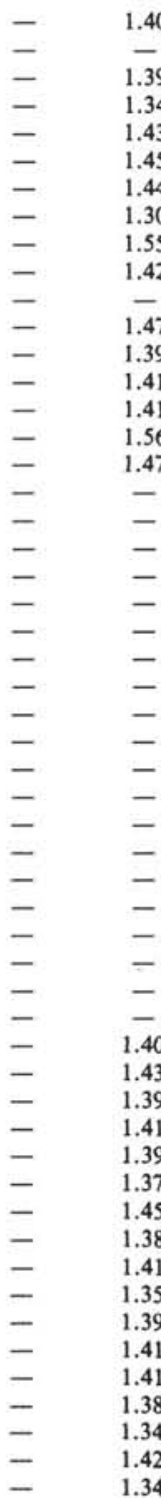

$\begin{array}{lll}1.40 & 1.24 & 203 \\ - & - & - \\ 1.39 & 1.21 & 232 \\ 1.34 & 1.19 & 25 \\ 1.43 & 1.30 & 157 \\ 1.45 & 1.27 & 176 \\ 1.44 & 1.26 & 18 \\ 1.30 & 1.24 & 199 \\ 1.55 & -\overline{1} \\ 1.42 & 1.28 & 172 \\ 1 . & 1.34 & 134 \\ 1.39 & 1.32 & 146 \\ 1.41 & 1.25 & 152 \\ 1.41 & 1.32 & 145 \\ 1.56 & 1.38 & 117 \\ 1.47 & 1.33 & 141 \\ - & - & - \\ - & - & - \\ - & - & - \\ - & 1.22 & 214 \\ - & - & - \\ - & - & - \\ - & - & - \\ - & - & - \\ - & - & - \\ - & - & - \\ - & - & - \\ - & - & - \\ - & - & - \\ - & - & - \\ - & - & - \\ - & - & - \\ - & - & - \\ 1.40 & - & - \\ 1.43 & 1.24 & 200 \\ 1.39 & 1.26 & 185 \\ 1.41 & 1.28 & 169 \\ 1.39 & 1.29 & 160 \\ 1.37 & 1.25 & 189 \\ 1.45 & 1.28 & 165 \\ 1.38 & 1.23 & 209 \\ 1.41 & 1.26 & 183 \\ 1.35 & 1.33 & 139 \\ 1.39 & 1.29 & 165 \\ 1.41 & 1.26 & 184 \\ 1.41 & 1.27 & 177 \\ 1.38 & 1.23 & 207 \\ 1.34 & 1.22 & 217 \\ 1.42 & 1.23 & 207 \\ 1.34 & 1.24 & 203 \\ & & \end{array}$

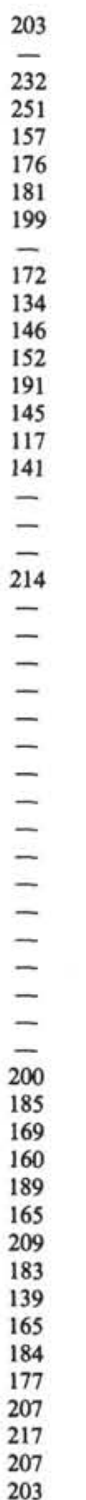

\begin{tabular}{|c|c|c|}
\hline 0.85 & 5.62 & 1.27 \\
\hline- & - & - \\
\hline 0.86 & 6.42 & 1.27 \\
\hline 0.87 & 6.94 & 1.25 \\
\hline 0.81 & 4.31 & 1.30 \\
\hline 0.83 & 4.88 & 1.27 \\
\hline 0.83 & 5.02 & 1.26 \\
\hline 0.85 & 5.51 & 1.25 \\
\hline- & - & - \\
\hline 0.83 & 4.75 & 1.28 \\
\hline 0.79 & 3.69 & 1.29 \\
\hline 0.80 & 4.04 & - \\
\hline 0.81 & 4.20 & 1.27 \\
\hline 0.84 & 5.29 & 1.27 \\
\hline 0.80 & 4.02 & 1.30 \\
\hline 0.76 & 3.23 & 1.31 \\
\hline 0.80 & 3.91 & 1.30 \\
\hline- & - & - \\
\hline- & - & - \\
\hline- & $\bar{s}$ & $\overline{1}$ \\
\hline 0.86 & 5.92 & 1.27 \\
\hline- & - & - \\
\hline- & - & - \\
\hline- & - & - \\
\hline- & - & - \\
\hline- & - & - \\
\hline- & - & - \\
\hline- & - & - \\
\hline- & - & - \\
\hline- & - & - \\
\hline- & - & - \\
\hline- & - & - \\
\hline- & - & - \\
\hline- & - & - \\
\hline- & - & - \\
\hline- & - & - \\
\hline $\bar{z}$ & $\overline{-}$ & - \\
\hline 0.72 & 5.53 & 1.28 \\
\hline 0.84 & 5.13 & 1.28 \\
\hline 0.82 & 4.69 & 1.28 \\
\hline 0.82 & 4.43 & 1.27 \\
\hline 0.84 & 5.24 & - \\
\hline 0.82 & 4.58 & 1.27 \\
\hline 0.85 & 5.78 & 1.28 \\
\hline 0.84 & 5.06 & 1.28 \\
\hline 0.79 & 3.84 & 1.27 \\
\hline 0.82 & 4.56 & 1.31 \\
\hline 0.84 & 5.10 & 1.28 \\
\hline 0.83 & 4.89 & 1.27 \\
\hline 0.85 & 5.73 & 1.27 \\
\hline 0.86 & 6.00 & 1.28 \\
\hline 0.85 & 5.72 & 1.27 \\
\hline & & \\
\hline
\end{tabular}

\begin{tabular}{|c|c|c|}
\hline 173 & 0.83 & 4.7 \\
\hline- & - & - \\
\hline 174 & 0.83 & 4.8 \\
\hline 193 & 0.84 & 5.3 \\
\hline 156 & 0.81 & $4.3 ?$ \\
\hline 175 & 0.83 & 4.8 \\
\hline 181 & 0.83 & 5.02 \\
\hline 195 & 0.84 & 5.4 \\
\hline- & - & - \\
\hline 169 & 0.82 & 4.7 \\
\hline 162 & 0.82 & 4.50 \\
\hline- & - & - \\
\hline 178 & 0.83 & 4.9 \\
\hline 177 & 0.83 & 4.92 \\
\hline 158 & 0.81 & 4.39 \\
\hline 152 & 0.81 & 4.22 \\
\hline 153 & 0.81 & 4.26 \\
\hline- & - & - \\
\hline- & - & - \\
\hline- & - & - \\
\hline 176 & 0.83 & 4.88 \\
\hline- & - & - \\
\hline- & - & - \\
\hline- & - & - \\
\hline- & - & - \\
\hline- & - & - \\
\hline- & - & - \\
\hline- & - & - \\
\hline- & - & - \\
\hline- & - & - \\
\hline- & - & - \\
\hline- & - & - \\
\hline- & - & - \\
\hline- & - & - \\
\hline- & - & - \\
\hline- & - & - \\
\hline- & - & - \\
\hline- & - & - \\
\hline 168 & 0.82 & 4.68 \\
\hline 168 & 0.82 & 4.65 \\
\hline 167 & 0.82 & 4.63 \\
\hline 173 & 0.83 & 4.79 \\
\hline- & - & - \\
\hline 174 & 0.83 & 4.82 \\
\hline 171 & 0.83 & 4.73 \\
\hline 168 & 0.82 & 4.65 \\
\hline 174 & 0.83 & 4.83 \\
\hline 149 & 0.81 & 4.14 \\
\hline 172 & 0.83 & 4.77 \\
\hline 178 & 0.83 & 4.94 \\
\hline 178 & 0.83 & 4.93 \\
\hline 169 & 0.82 & 4.68 \\
\hline 178 & 0.83 & 4.93 \\
\hline 154 & 0.81 & 4.27 \\
\hline
\end{tabular}

Hole 581

$2-6,107-129$ 
Table 1. (Continued).

\begin{tabular}{|c|c|c|c|c|c|c|c|c|c|c|c|c|c|c|}
\hline \multirow{3}{*}{$\begin{array}{l}\text { Core-Section } \\
\text { (interval or } \\
\text { level in } \mathrm{cm} \text { ) }\end{array}$} & \multirow{3}{*}{$\begin{array}{l}\text { Av. } \\
\text { sbd } \\
\text { (m) }\end{array}$} & \multicolumn{9}{|c|}{ Shipboard } & \multicolumn{4}{|c|}{ Laboratory } \\
\hline & & \multicolumn{2}{|c|}{ Shear strength } & \multirow{2}{*}{$\begin{array}{l}\text { P-wave } \\
\text { velocity } \\
(\mathrm{m} / \mathrm{s})\end{array}$} & \multirow{2}{*}{$\begin{array}{l}\text { S-wave } \\
\text { velocity } \\
(\mathrm{m} / \mathrm{s})\end{array}$} & \multirow{2}{*}{$\begin{array}{c}\text { GRAPE } \\
\text { bulk } \\
\text { density } \\
\left(\mathrm{g} / \mathrm{cm}^{3}\right)\end{array}$} & \multirow{2}{*}{$\begin{array}{l}\text { Bulk } \\
\text { density } \\
\left(\mathrm{g} / \mathrm{cm}^{3}\right)\end{array}$} & \multirow{2}{*}{$\begin{array}{l}\text { Water } \\
\text { content } \\
(\%)\end{array}$} & \multirow[b]{2}{*}{ Porosity } & \multirow[b]{2}{*}{$\begin{array}{l}\text { Void } \\
\text { ratio }\end{array}$} & \multirow{2}{*}{$\begin{array}{l}\text { Bulk } \\
\text { density } \\
\left(\mathrm{g} / \mathrm{cm}^{3}\right)\end{array}$} & \multirow{2}{*}{$\begin{array}{l}\text { Water } \\
\text { content } \\
(\%)\end{array}$} & \multirow[b]{2}{*}{ Porosity } & \multirow[b]{2}{*}{$\begin{array}{l}\text { Void } \\
\text { ratio }\end{array}$} \\
\hline & & $\begin{array}{l}\text { Torvane } \\
\quad\left(\mathrm{g} / \mathrm{cm}^{2}\right.\end{array}$ & 2) W.F. & & & & & & & & & & & \\
\hline \multicolumn{15}{|l|}{ Hole 581 (Cont.) } \\
\hline $3-5,124-135$ & 198.30 & 200 & 221 & 1510 & - & 1.39 & 1.24 & 200 & 0.85 & 5.52 & 1.26 & 184 & 0.84 & 5.11 \\
\hline $4-2,60-70$ & 202.65 & 600 & 692 & 1509 & - & 1.34 & 1.23 & 205 & 0.85 & 5.66 & 1.27 & 174 & 0.83 & 4.84 \\
\hline $4-2,122$ & 203.22 & - & - & 1514 & - & - & - & - & - & - & - & - & - & - \\
\hline $6-2,95-114$ & 222.05 & 1025 & 744 & 1513 & - & 1.41 & 1.28 & 171 & 0.83 & 4.72 & 1.25 & 187 & 0.84 & 5.18 \\
\hline $6-5,66-86$ & 226.26 & 1275 & 640 & 1501 & - & 1.39 & 1.31 & 148 & 0.80 & 4.10 & 1.29 & 162 & 0.82 & 4.50 \\
\hline $7-5,60-78$ & 235.69 & 750 & 655 & 1519 & - & 1.43 & 1.30 & 155 & 0.81 & 4.28 & 1.28 & 165 & 0.82 & 4.58 \\
\hline $8-5,11-100$ & 245.06 & 1225 & 699 & 1484 & - & 1.56 & 1.39 & 115 & 0.76 & 3.18 & 1.29 & 161 & 0.82 & 4.46 \\
\hline $8-5,95$ & 245.45 & - & - & 1478 & - & - & - & - & - & - & - & - & - & - \\
\hline $9-4,45-63$ & 253.04 & 1038 & 780 & 1472 & - & 1.60 & 1.49 & 85 & 0.70 & 2.35 & 1.31 & 152 & 0.81 & 4.23 \\
\hline $10-3,100-114$ & 261.57 & 913 & 1156 & 1474 & - & 1.63 & 1.54 & 75 & 0.67 & 2.08 & 1.32 & 146 & 0.80 & 4.06 \\
\hline
\end{tabular}

Note: See Results for further explanation of column heads. - means not measured.

the effective normal stress has been reduced to nearly zero by the sampling process. It is not surprising, therefore, that the shear strength as measured by the vane is higher in clay sediments, which have higher values of cohesion. In addition, it is thought that the more brittle nature of the interparticle bonds in the carbonates compared with the clays will result in more structural disturbance during coring and recovery.

Two interesting aspects of the shear wave velocities should be mentioned here. First, measurements made parallel and perpendicular to the core axis indicate a total lack of anisotropy within any of the lithologies. Second, the large increase in both the shear strength and the shear wave velocities in the brown clay below $65 \mathrm{~m}$ (while interbedded with carbonates) may be indicative of the onset of diagenesis. The shear strength measurements also often display a systematic decrease in strength between the bottom of one core and the top of the next. This occurred even though the sediments appeared undisturbed. It is thought that this may reflect a decreasing disturbance factor for clays down the length of each hydraulic piston core. A "flow-in" section exists at around $30-38 \mathrm{~m}$ sub-bottom depth in Hole 576, which unfortunately coincides with a highly disturbed region in Hole 576B. Shear strength values in this section are much reduced (a factor of 1/2) indicating an in situ remolding sensitivity of 2 . The shear wave velocity is also reduced in this section, but not as prominently.

\section{Site $\mathbf{5 7 7}$}

This site consists of about $120 \mathrm{~m}$ of nannofossil oozes which were divided into three lithologic units based on color changes (see Site 577 chapter, this volume). Physical property data for this site are presented for Holes 577 and 577A in Table 1 . These data are presented graphically in Figure 4.

The water content profile shows some fluctuations near the surface but generally decreases from around 90 to $75 \%$ at $60 \mathrm{~m}$. At this point there is a dramatic decrease to around $50 \%$ which is maintained down to $120 \mathrm{~m}$. Note that the GRAPE bulk density does not show this important discontinuity in the data. A similar dis- continuity is exhibited at $60 \mathrm{~m}$ in both the compressional wave velocity profile and in the shear strength profile; however, it is not clearly defined in the shear wave velocity profile. The compressional wave velocity fluctuates around $1475 \mathrm{~m} / \mathrm{s}$ down to $60 \mathrm{~m}$ and then increases to around $1525 \mathrm{~m} / \mathrm{s}$ for the remaining length of the holes with a few high-velocity layers around $1600 \mathrm{~m} / \mathrm{s}$. The shear strength profile exhibits a great deal of fluctuation down to $60 \mathrm{~m}$ with values ranging up to $600 \mathrm{~g} / \mathrm{cm}^{2}$. The shear strength is very low from $60 \mathrm{~m}$ down to the bottom of the holes at $120 \mathrm{~m}$ with values around $100 \mathrm{~g} /$ $\mathrm{cm}^{2}$. For this site there was a distinct difference between values obtained from the hand-held Torvane and from the motorized vane, with the Torvane giving much higher readings. The shear wave velocity profile shows no definite trend and fluctuates around $50 \mathrm{~m} / \mathrm{s}$ throughout the hole. Poor signal quality in most of these cores limits the accuracy of these measurements to $\pm 10 \mathrm{~m} / \mathrm{s}$.

The discontinuity exhibited by the water content, compressional wave velocity, and shear strength profiles at $60 \mathrm{~m}$ occurs just below the boundary between lithologic Units I and II. It corresponds to a possible $30 \mathrm{~m}$.y. missing section in the mid-Tertiary (see Site 577 chapter, this volume) which, at sedimentation rates of $3.0 \mathrm{~m} / \mathrm{m}$.y., would represent $90 \mathrm{~m}$ of missing sediment. The rapid change in water content suggests that this may have been an erosional event because the material may be overconsolidated. However, the subtle (but distinct) increase in carbonate content across the boundary could mean that the different water contents are simply a function of the different types of skeletal material; in which case it could be argued that the hiatus was a nondepositional event. The increase in compressional wave velocity at the discontinuity is consistent with the decrease in water content. However, the decrease in shear strength is not so easily explained. It is suggested that the relatively subtle change in carbonate content from $90 \%$ above the boundary to $95 \%$ below is enough to reduce drastically the strength as a result of increased coring disturbance caused by an increase in the brittle nature of the bonds. Alternatively, it may simply reflect the change in cohesion caused by the small reduction in clay content. This is an 

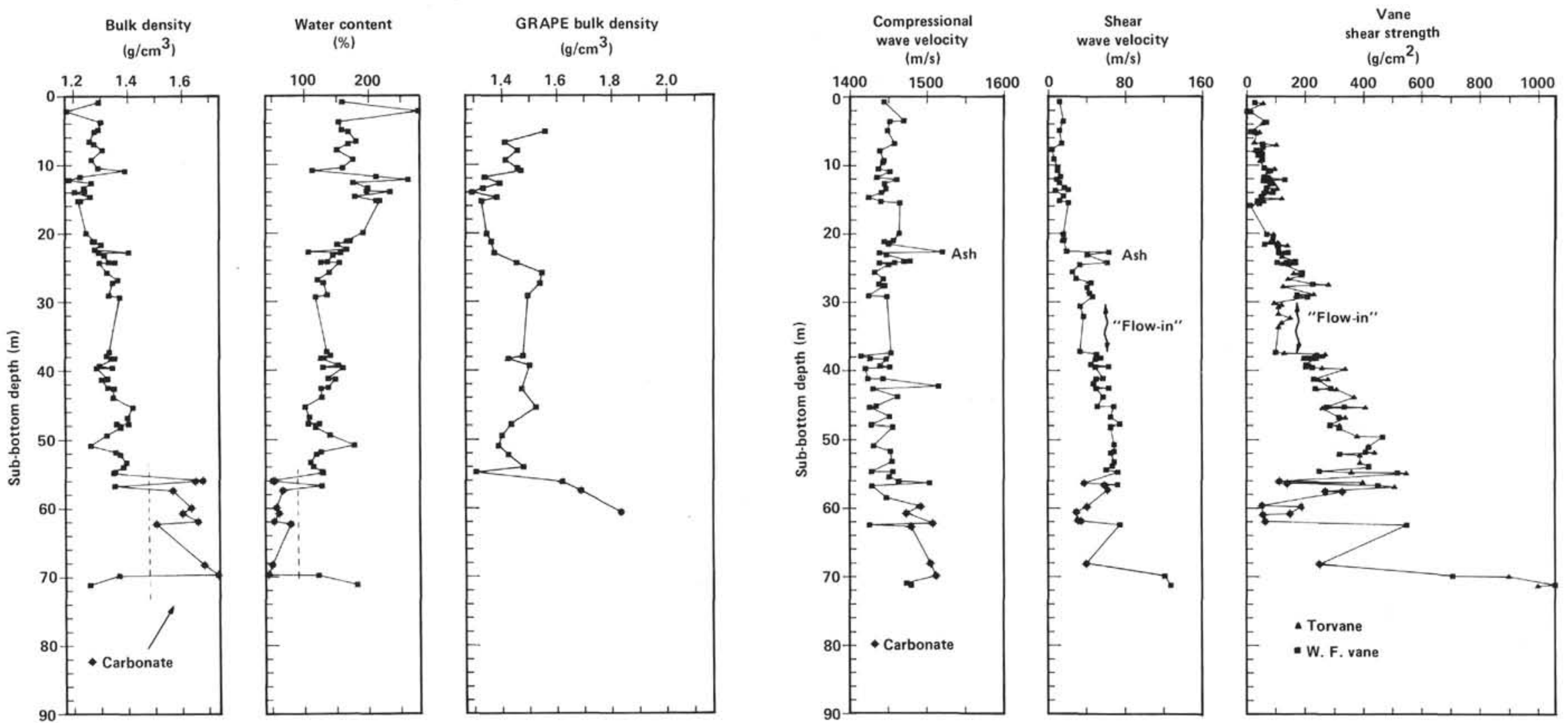

Figure 3. Gravimetric, GRAPE, velocity, and shear strength data for Site 576 (Holes 576 and 576B). 

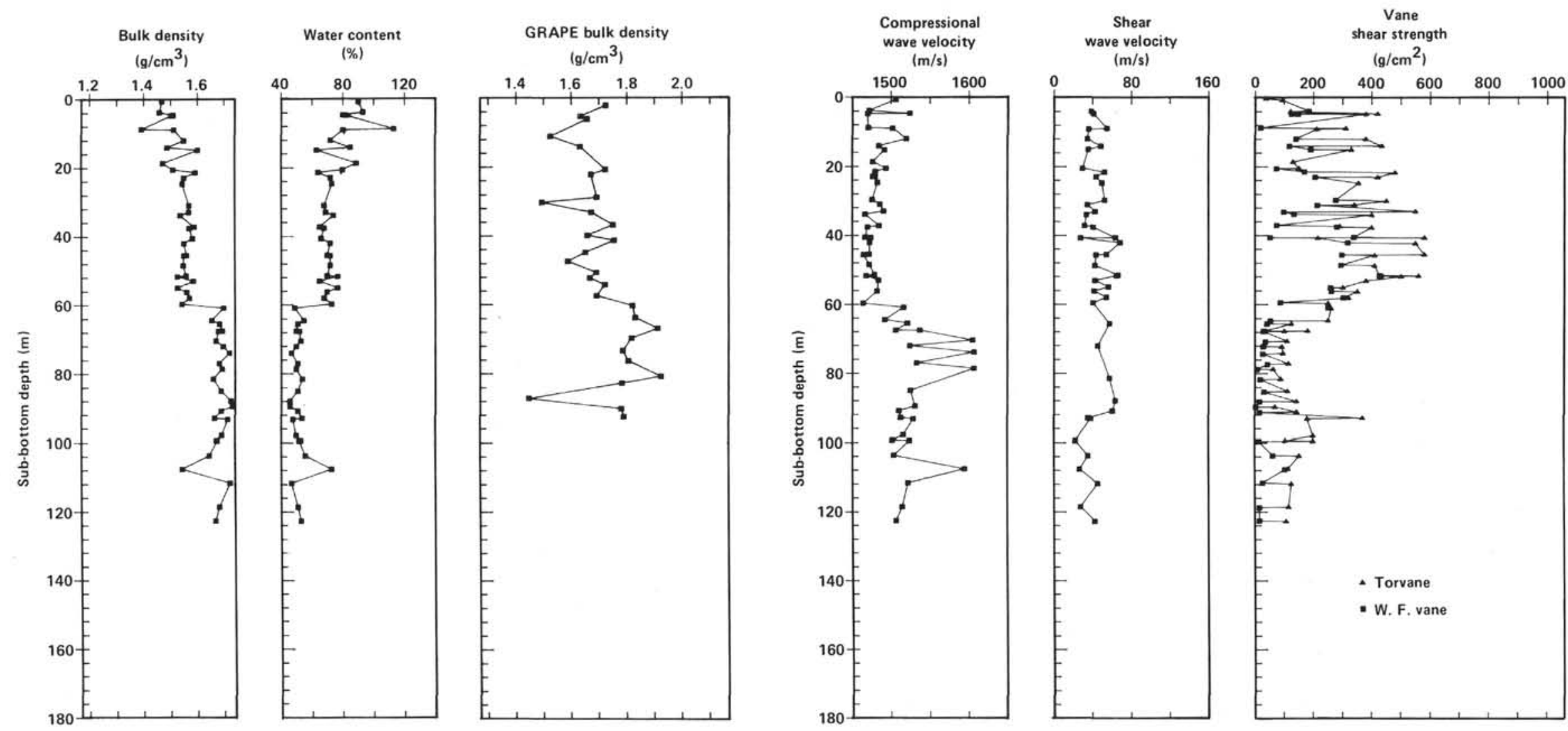

Figure 4. Gravimetric, GRAPE, velocity, and shear strength data for Site 577 (Holes 577 and 577A) 
extension of the argument presented to explain the differences reported between clays and carbonates for Site 576.

\section{Site 578}

This site consists of a thick section of siliceous clays, (lithologic Units I and II) down to $124.5 \mathrm{~m}$, below which a dark brown pelagic clay extends to $176 \mathrm{~m}$ (lithologic Unit III) (see Site 578 chapter, this volume). Within the siliceous clays numerous pyrite-cemented layers and ash layers occur. The data for Site 578 are presented in Table 1 and in Figure 5.

Some fluctuations occur in the water content profile but, generally, the water content decreases from around $175 \%$ near the surface to around $125 \%$ at the base of lithologic Unit II (124.5 m). Below this, the water content in the dark brown clay decreases more rapidly until at $170 \mathrm{~m}$ it is around $70 \%$. The GRAPE bulk density profile shows a major discontinuity at around $120 \mathrm{~m}$, which illustrates the sensitivity of the measurement to the mineral attenuation coefficient. The compressional wave profile is dominated by high-velocity layers of pyrite-indurated clay and ash beds superimposed on a constant velocity profile (around $1469 \mathrm{~m} / \mathrm{s}$ ) down to $160 \mathrm{~m}$ (Fig. 5). Only a few of the numerous higher velocity layers were measured but it can be assumed that they all have much higher velocities (between 1600 and $1800 \mathrm{~m} / \mathrm{s}$ ). This corresponds (where measurements were taken) to higher bulk densities (Fig. 5). Below $160 \mathrm{~m}$ there is some indication that a positive velocity gradient is developing in the very dark pelagic clay which is not consistent with an increase in bulk density. This may represent an early stage of diagenesis.

Shear wave velocities increase slowly from $40 \mathrm{~m} / \mathrm{s}$ near the surface to $65 \mathrm{~m} / \mathrm{s}$ at a depth of $120 \mathrm{~m}$ (Fig. 5). A rapid increase occurs between lithologic Units II and III with velocities reaching around $150 \mathrm{~m} / \mathrm{s}$ at $170 \mathrm{~m}$ in the dark brown pelagic clay. Shear strength values exhibit some scatter but generally increase from less than $100 \mathrm{~g} / \mathrm{cm}^{2}$ near the surface to about $600 \mathrm{~g} / \mathrm{cm}^{2}$ at the base of lithologic Unit II (124.5 m). Below this, the value jumps to around $1000 \mathrm{~g} / \mathrm{cm}^{2}$ and increases to above $1600 \mathrm{~g} / \mathrm{cm}^{2}$ at $170 \mathrm{~m}$ in the dark brown pelagic clay. Recovery from hydraulically pistoned Cores $17,18,19$, and 20 in the brown clay were progressively poorer, with Core 20 being only $0.81 \mathrm{~m}$ long. Shear strengths at around $1700 \mathrm{~g} / \mathrm{cm}^{2}$ in these pelagic brown clays obviously represent the operational limits of the HPC in its present configuration. It is also interesting to note that no flowin occurred in these cores. Presumably, water must have flowed around the piston during pull-out as a result of the high lateral stresses plugging the core barrel.

\section{Site 579}

A thick section of siliceous clay with numerous ash and thin indurated layers occurs at this site. This single lithologic unit was divided into three subunits: a siliceous clay $(0-60 \mathrm{~m})$, a clayey siliceous ooze $(60-102.5 \mathrm{~m})$, and a clayey diatom ooze (102.5-149.5 m) (see Site 579 chapter, this volume). The data for this site are presented in Table 1 and in Figure 6.
The water content profile (Fig. 6) can be split into three units. Unit I $(0-42 \mathrm{~m})$ shows a decreasing water content from around 230 to $110 \%$ which may be the result of normal consolidation processes. There is a discontinuity between 40 and $42 \mathrm{~m}$, where the water content suddenly increases from 110 to $175 \%$. Unit II (42$134 \mathrm{~m}$ ) exhibits an increased water content downcore from 175 to $228 \%$. Below this, Unit III (134-149 m) shows the water content decreasing to $158 \%$. These units do not correlate with any of the lithological subunits based on visual inspection and smear slide analyses. However, it could be inferred that the increase in water content from 40 to $134 \mathrm{~m}$ correlates with an increase in the siliceous microfossil content.

The compressional wave velocity profile (Fig. 6) is dominated by many (61) high-velocity ash layers and numerous $(\sim 325)$, thin $(<0.5 \mathrm{~cm})$, stiff-to-indurated, dark greenish grey layers. Velocities measured in a few ash layers are typically about $1600 \mathrm{~m} / \mathrm{s}$. The thin, indurated, green layers also have higher velocities (these values are not shown in Fig. 6) but they tend to be variable (one was as high as $1770 \mathrm{~m} / \mathrm{s}$ ). Velocities in the siliceous clay and ooze are remarkably uniform throughout the site at around $1480 \mathrm{~m} / \mathrm{s}$.

Shear wave velocity measurements (Fig. 6) were prematurely terminated at this site as one of the transducers broke on insertion. Despite being pushed into some very stiff sediments a large number of times, the somewhat fragile design had proved satisfactory until this time. The data obtained between 18 and $73 \mathrm{~m}$ show no distinct trends with depth, the velocity remaining nearly constant at around $45 \mathrm{~m} / \mathrm{s}$.

Shear strength measurements, although exhibiting a large amount of scatter, show increasing strength down the hole (Fig. 6). A major discontinuity occurs at $95 \mathrm{~m}$ with very high shear strengths below (up to $1000 \mathrm{~g} / \mathrm{cm}^{2}$ ) compared with shear strengths of about $400 \mathrm{~g} / \mathrm{cm}^{2}$ in shallower sections. This discontinuity does not correlate with any of the lithologic changes. A good example of "flow-in" is found at $68 \mathrm{~m}$ (the bottom of Section 579A-6-5). Measurement with both the hand-held and motorized vane shows that the remolded "flow-in" section had decreased in strength from $380 \mathrm{~g} / \mathrm{cm}^{2}$ in the "undisturbed" region to $100 \mathrm{~g} / \mathrm{cm}^{2}$ in the section containing "flow-in." This provides an in situ remolding sensitivity of about 4 . A small reduction in the compressional wave velocity can also be seen at this point, although the remolding did not change the water content.

\section{Site $\mathbf{5 8 0}$}

The sediments cored at Site 580 are similar to those cored at Site 579 and consist of a thick section of siliceous clays with a large number of volcanic ash layers (89) and numerous, thin, stiff-to-indurated, dark greenish-grey layers (see Site 580 chapter, this volume). The data for this site are presented in Table 1 and in Figure 7.

The water content profile shows a remarkably high and constant value $(\sim 175 \%)$ throughout the entire hole with no variation resulting from changing microfossil content (Fig. 7). As at Sites 578 and 579, the compressional wave velocity profile is dominated by the high ve- 

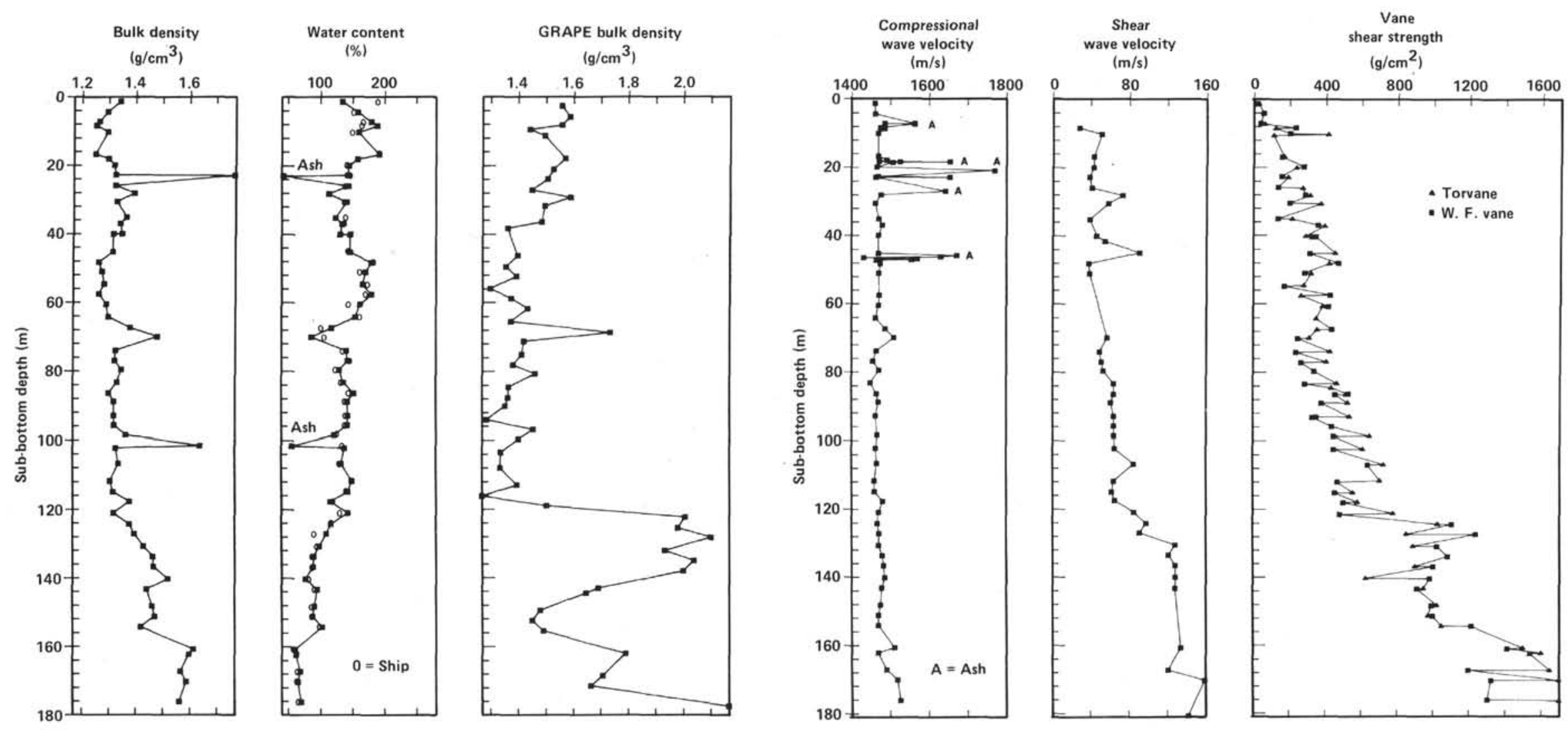

Figure 5. Gravimetric, GRAPE, velocity, and shear strength data for Site 578. 

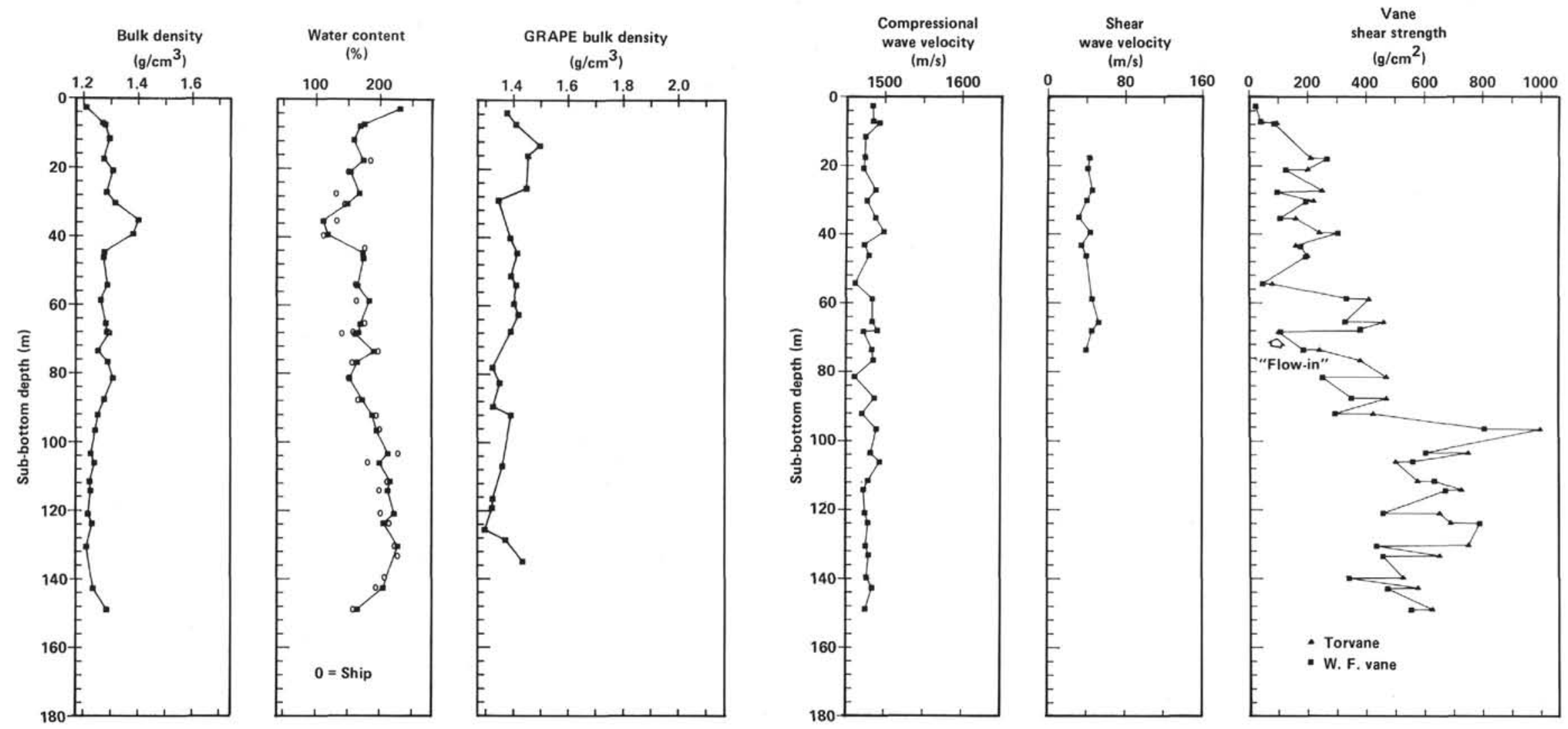

Figure 6. Gravimetric, GRAPE, velocity, and shear strength data for Site 579 (Holes 579 and 579A). 
locity, indurated clay and ash layers. Only two of the ash layers are shown in Figure 7. Velocities in the siliceous clays and oozes rise gradually downhole from about $1470 \mathrm{~m} / \mathrm{s}$ at the surface to about $1490 \mathrm{~m} / \mathrm{s}$ at a depth of $150 \mathrm{~m}$. The shear strength measurements show a nearly linear increase with depth ranging from only 20 $\mathrm{g} / \mathrm{cm}^{2}$ near the surface to over $800 \mathrm{~g} / \mathrm{cm}^{2}$ at $150 \mathrm{~m}$ (Fig. 7).

\section{Site 581}

Limited time restricted the coring program at this site. Consequently, sediments were only recovered between 181.5 and $276.6 \mathrm{~m}$ using the rotary core (see Site 581 chapter, this volume). Lithologic Unit I extends from 181.5 to $245 \mathrm{~m}$ and consists of a biosiliceous clay with some ash and indurated layers. This unit is similar to the siliceous clay found at Sites 579 and 580 . Lithologic Unit II is a brown to dark brown pelagic clay similar and probably of comparable age to that found at Sites 576 and 578 .

The physical properties data for Site 581 are presented in Table 1 and in Figure 8. The water content profile shows a decrease from $229 \%$ at $190 \mathrm{~m}$ to $146 \%$ at $261 \mathrm{~m}$ (Fig. 8). It should be noted that there is a large discrepancy toward the bottom of the hole between the shipboard data and the laboratory data that will be discussed later. Compressional wave velocity measurements show a constant profile in the siliceous clay unit at around $1505 \mathrm{~m} / \mathrm{s}$ but this decreases to $1475 \mathrm{~m} / \mathrm{s}$ in the brown pelagic clay (Fig. 8). Some compressional wave measurements were made on selected samples of chert and basalt (lithologic Units III and IV) and these varied between 4000 and $5500 \mathrm{~m} / \mathrm{s}$ and are not shown in Figure 8 .

The shear strength measurements (Fig. 8) should be viewed with even more caution than usual. Rotary coring causes significantly more structural disturbance than the HPC. This is true even when the stratigraphic sequence looks undisturbed. Much of the material in Cores 1-7 consists of alternating layers of soft and stiff sediments, a pattern that is probably caused by intermittent "rotation" and "punch" coring (rotation causing the most disturbance and hence the softer layers). Measurement sites were carefully selected in the largest sections of stiff material that could be found in each core. Even in the stiff sediments the shear strength measurements were hampered by the brittle nature of the semi-indurated sediments. This resulted in major cracks developing either upon initial insertion of the vane or during the test. The test is not valid unless the failure plane is cylindrical. In an attempt to overcome this problem, a slight pressure was applied to either side of the split core by using two strips of aluminium held together by G-clamps. This eliminated the gap between the liner and the sediment and prevented the major cracking.

The first few shear strength readings (between 190 and $210 \mathrm{~m}$ ) were taken without the G-clamp modification and show low shear strengths $\left(200-600 \mathrm{~g} / \mathrm{cm}^{2}\right)$ because of cracking. All of the remaining measurements were made with the G-clamp and show higher but fluc- tuating values around $1000 \mathrm{~g} / \mathrm{cm}^{2}$. These values are not as high as those found in the similar dark brown pelagic clays at Site $578\left(>1600 \mathrm{~g} / \mathrm{cm}^{2}\right)$ despite being subjected to a much higher overburden stress. It is probable that these lower values can be mainly attributed to increased disturbance at Site 581 caused by the rotary drilling process.

\section{DISCUSSION}

\section{Effective Stress Profiles}

From the bulk density data, the vertical effective stress or overburden stress can be calculated down each hole. The total vertical pressure or effective stress $(P)$ is given by

$$
P=\Sigma(\gamma \Delta z)
$$

where $\Delta z$ is the incremental depth and $\gamma$ is the submerged unit weight. Calculating $\gamma$ from the bulk density, effective stress profiles for all six sites have been plotted in Figure 9. The position of the profile for Site 581 has been estimated assuming the upper sediments were similar to Sites 579 and 580 . Figure 9 illustrates the large differences in effective stress character between the different sediment types. Site 577 has the smallest gradient caused by the most dense carbonate sediments (i.e., effective stress increases rapidly with sub-bottom depth). It also shows the change in gradient at $60 \mathrm{~m}$ where the mid-Tertiary hiatus occurs. Site 576 shows a decrease in gradient below $55 \mathrm{~m}$ where the sediment increases in density (brown clay to carbonate ooze). The siliceous clays at Sites 579 and 580 are very similar showing a complete lack of any significant consolidation throughout $150 \mathrm{~m}$. At Site 581 , the decreasing gradient indicates that some consolidation is occurring when the depth approaches $250 \mathrm{~m}$.

\section{Water Content}

There is some discrepancy between the values of water content calculated from the shipboard measurements and those from the laboratory measurements. For Sites $578,579,580$, and 581 , the shipboard measurements have been plotted alongside the shore-based laboratory measurements (Figs. 5, 6, 7, and 8, respectively). For Sites 576 and 577 , the shipboard measurements are almost identical to the laboratory measurements (see Table 1), hence only the laboratory values have been plotted. This situation is similar for Sites 578 and 579 (see Figs. 5 and 6). However, the discrepancy arises for Sites 580 and 581 (see Figs. 7 and 8). For Site 580, the shipboard measurements show approximately the same values as the laboratory measurements but they exhibit more scatter (Fig. 7). For Site 581 , the shipboard measurements below 240 $\mathrm{m}$ are substantially less than the laboratory values (Fig. 8 ). The only variable that can be attributed to these differences is ship motion and the resulting inaccuracy of the shipboard weighings on the triple beam balance. Ship motion during Leg 86 was marginally worse during op- 

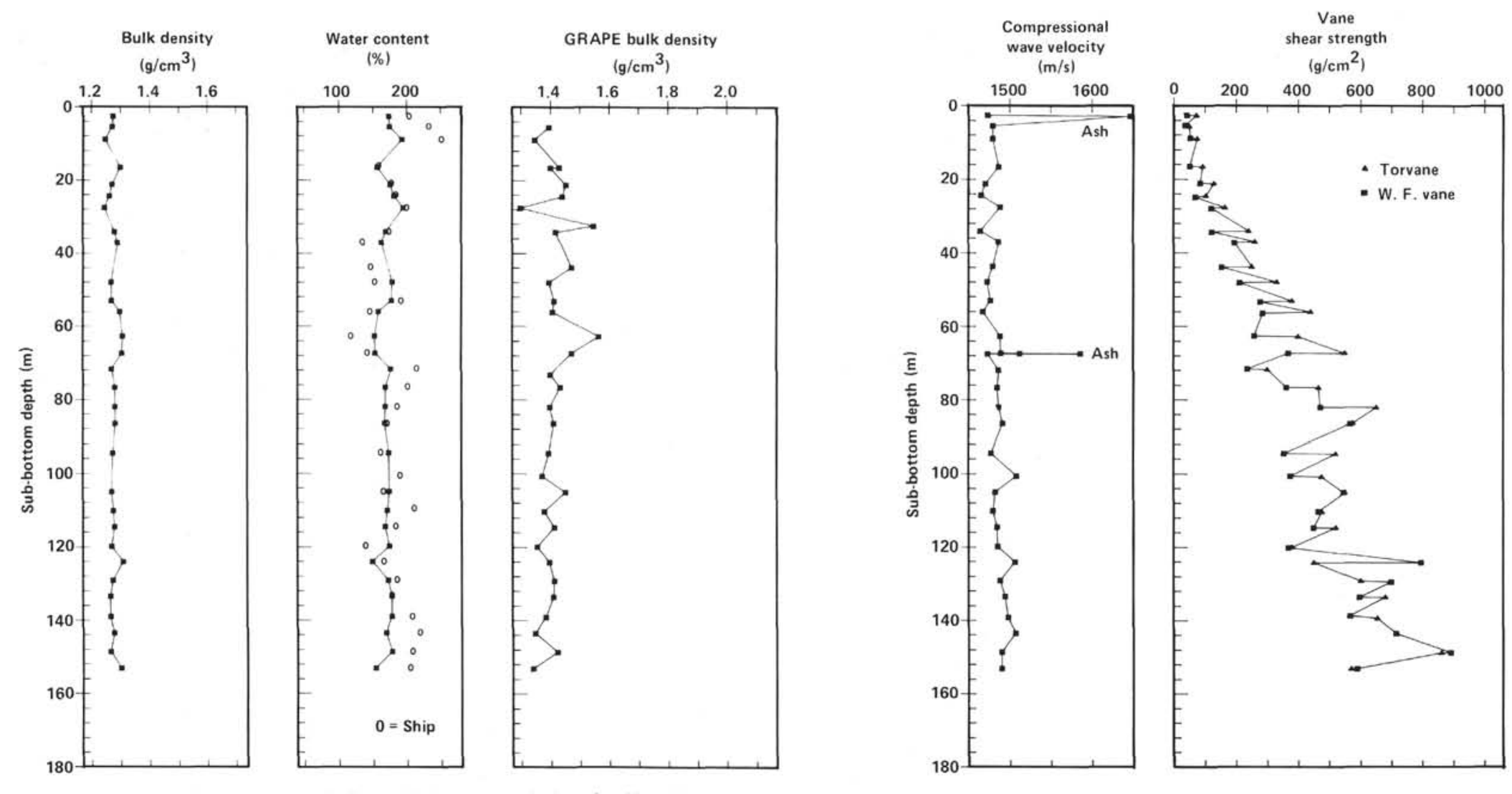

Figure 7. Gravimetric, GRAPE, velocity, and shear strength data for Site 580. 

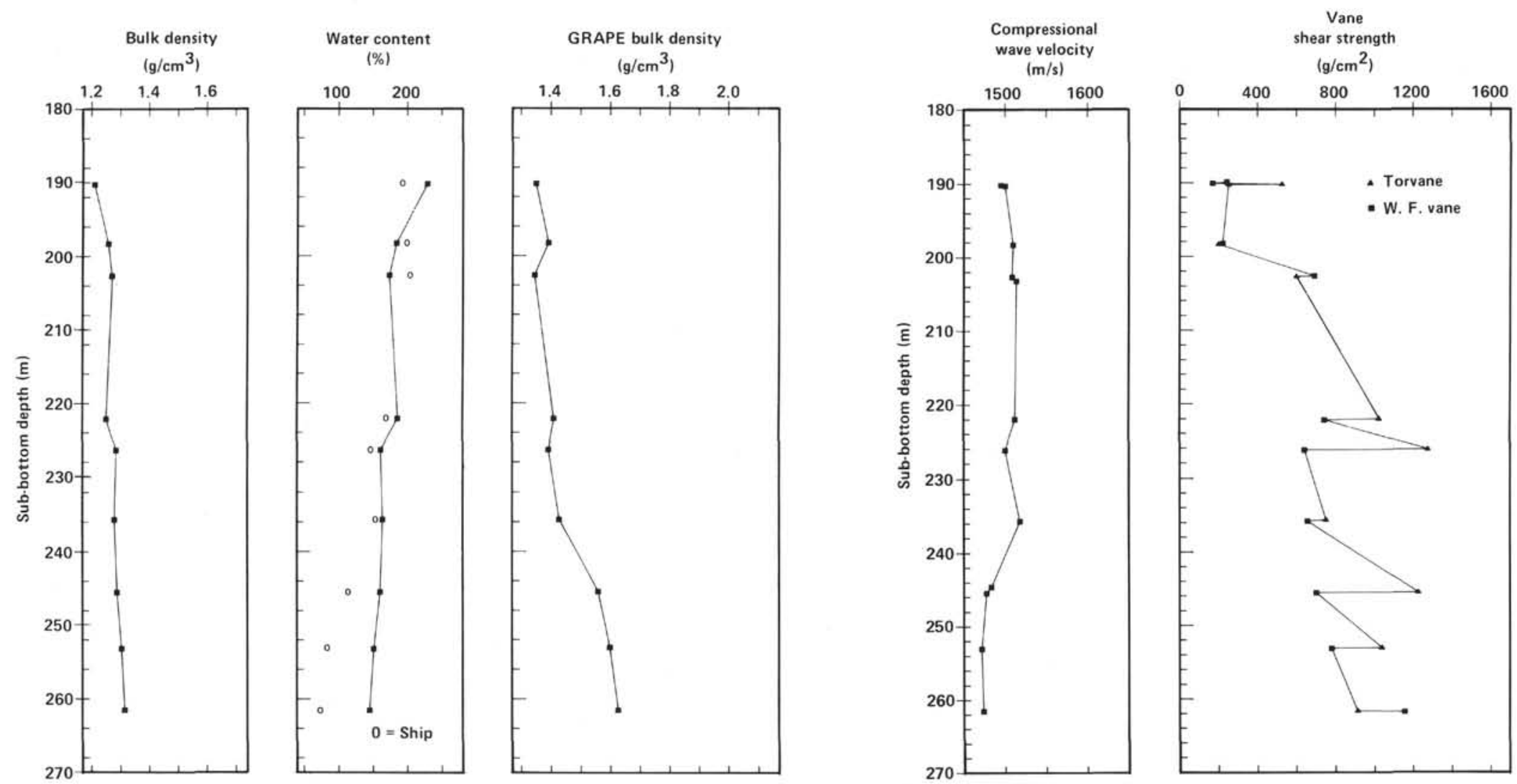

Figure 8. Gravimetric, GRAPE, velocity, and shear strength data for Site 581 


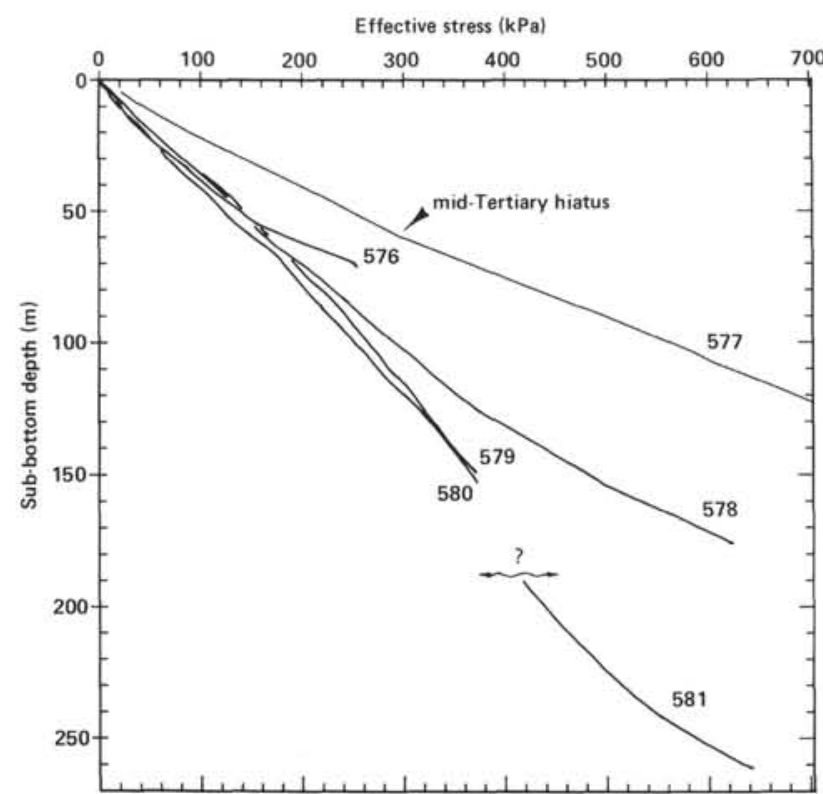

Figure 9. Effective stress (overburden stress) versus depth, calculated from the bulk density profiles for Sites 576-581.

erations at Sites 580 and 581 compared with the earlier sites, consequently the shore-based laboratory values are considered to be the most reliable.

\section{Shear Strength and Shear Wave Velocities}

Two sets of shear strength measurements (one using the hand-held Torvane and one using the motorized Wykeham Farrance vane) were conducted throughout the leg. The Torvane measurement is normally considered to provide a much more approximate estimate of undrained shear strength than the more controlled motorized vane. It is interesting, therefore, to examine whether there is any consistent difference between the two devices in different sediment types. Figure 10 shows a plot of Torvane shear strength versus vane shear strength (W.F. motorized vane) for all of the Leg 86 sediments. It is clear that, despite a significant amount of scatter, the overall trend from Figure 10 shows the Torvane shear strength $\left(T_{\mathrm{u}}\right)$ to be greater, on average, than the W.F. motorized vane shear strength $\left(M_{\mathrm{u}}\right)$ where $T_{\mathrm{u}}=1.13 M_{\mathrm{u}}$. In the carbonate sediments at Site 577 , the difference between $M_{\mathrm{u}}$ and $T_{\mathrm{u}}$ was even greater than in the brown clays and siliceous clays at the other sites. For these carbonates, $T_{\mathrm{u}} \simeq 2 M_{\mathrm{u}}$.

Shear wave velocities ranged from only a few meters per second to over $150 \mathrm{~m} / \mathrm{s}$ and correlated approximately with the shear strength data. Figure 11 is a scatter plot of the Torvane strength versus the shear wave velocity for all the data.

\section{Consolidation and Permeability Profiles of Red Clay}

Whole core samples of red clay from Sites 576 and 578 were tested to determine their consolidation and permeability characteristics. The back-pressurized consolidation/permeability technique used is described by Schultheiss and Gunn (1985). The data are plotted in Figures 12 and 13 . Figure 12 shows the data for the individual samples tested (void ratio, vertical effective stress, and permeability relationships). Figure 13 shows the permeability profile through the red clay (permeabilities are taken from the individual plots at the calculated overburden stress) and the overconsolidation ratio profile. It can be seen that the permeability-void ratio relationship lies within a narrow band ranging from $10^{-4} \mathrm{~mm} / \mathrm{s}$ at a void ratio of 6 down to $5 \times 10^{-9} \mathrm{~mm} / \mathrm{s}$ at a void ratio of 1 . The permeability, therefore, decreases rapidly in the first $40 \mathrm{~m}$ of sediment (two-and-a-half orders of magnitude) but relatively little (only another order of magnitude) at a sub-bottom depth of $170 \mathrm{~m}$.

The overconsolidation ratio reveals essentially normal consolidation below a depth of $20 \mathrm{~m}$ but with considerable "apparent overconsolidation" between 0 and $20 \mathrm{~m}$. The Hole 576A data are included in the large geotechnical data set reported and fully discussed by the Geotechnical Consortium (this volume). The purpose of including it here is to show how the data from the deep red clay found at Site 578 fit into the overall pattern.

\section{CONCLUSIONS}

Leg 86 of the Deep Sea Drilling Project provided HPC cores of red clays, carbonate oozes, and siliceous clays from the Northwest Pacific. Some physical property measurements were made on these sediments (bulk density, water content, shear strength, compressional wave and shear wave velocity, consolidation, and permeability) which illustrate the behavior of the different sediment types. Progressive consolidation is observed in the water content profiles of the red clay and carbonate ooze (Sites 576 and 577) but little if any progressive consolidation is observed in the thick sequence of siliceous clays at Sites 579 and 580. Despite this lack of consolidation at Sites 579 and 580 , the vane shear strength increases progressively downhole. The compressional wave profiles are similar in nature to the water content profiles illustrating that, in unconsolidated sediments, the velocity is essentially a function of the bulk density. The high water contents at all the sites, apart from Site 577, produced compressional wave velocities lower than water velocities except in the ash and pyrite-indurated layers. In these numerous layers the velocity was higher but only a few measurements were made. It would be a significant improvement if compressional wave velocity were logged continuously and automatically, similar to the GRAPE technique. In this way detailed velocity profiles could be obtained, enabling the seismic stratigraphy to be examined in detail and correlated with seismic reflection profiles.

The red clays exhibit a significant amount of "apparent" overconsolidation in the upper $20 \mathrm{~m}$. Below this depth, they behave normally. Permeabilities in the red clays fall by three-and-a-half orders of magnitude in the top $170 \mathrm{~m}$ as the void ratio decreases.

The discrepancy between shipboard and shore-based laboratory determinations of water content was found to correlate with ship motion. It was concluded that laboratory water contents were the most reliable. The GRAPE bulk density determinations do not reveal important changes in bulk density. In fact, the GRAPE bulk densi- 


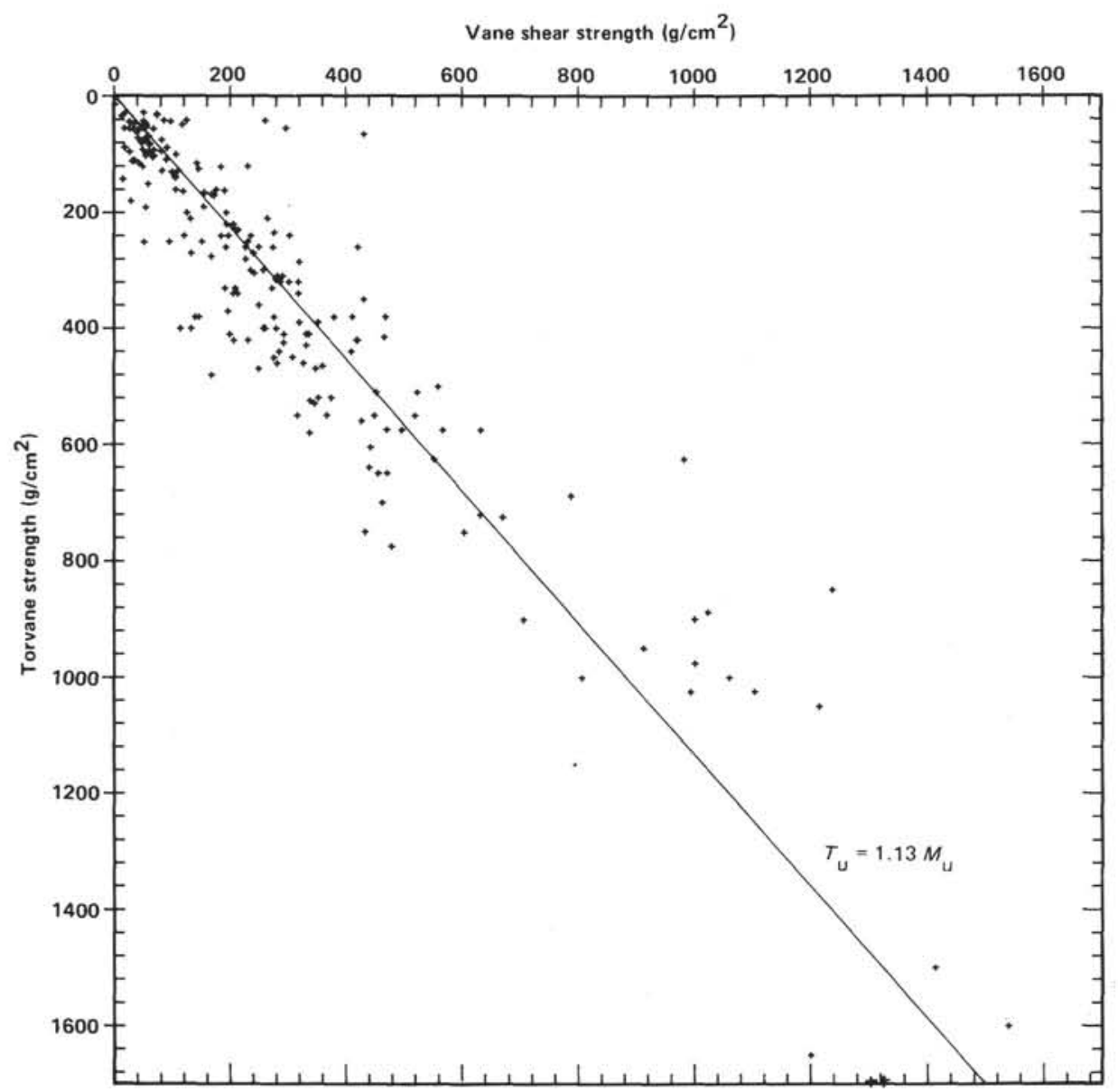

Figure 10. Torvane strength $\left(T_{u}\right)$ versus vane shear strength $\left(M_{u}\right)$ (W.F. motorized vane) for all the sediments from Leg 86 .

ty profiles for all the sites correlate very poorly with the reliable laboratory-determined values of bulk density.

\section{ACKNOWLEDGMENTS}

The author would like to thank Larry Mayer and Bill Levy for reviewing this paper and for providing some constructive comments. I would also like to thank Tom Janecek, who helped with some of the on board measurements.

\section{REFERENCES}

Bennett, R. H., and Keller, G. H., 1973. Physical properties evaluation. In Van Andel, T. H., Heath, G. R., et al., Init. Repts. DSDP, 16: Washington (U.S. Govt. Printing Office), 513-520.

Boyce, R. E., 1973. Appendix I: Physical property methods. In Edgar, N. T., Saunders, J. B., et al., Init. Repts. DSDP, 15: Washington (U.S. Govt. Printing Office), 1115-1128.

, 1976. Appendix 1: Definitions and laboratory techniques of compressional sound velocity parameters and wet-water content, wet-bulk density and porosity parameters by gravimetric and gamma ray attenuation techniques. In Schlanger, S. O., Jackson, E. D., et al., Init. Repts. DSDP, 33: Washington (U.S. Govt. Printing Office), 931-958.

1977. Deep Sea Drilling Project procedures for shear strength measurement of clayey sediment using modified Wykeham Farrance laboratory vane apparatus. In Barker, P., Dalziel, I. W. D., et al., Init. Repts. DSDP, 36: Washington (U.S. Govt. Printing Office), 1059-1068.

Curray, J. R., Moore, D. G., et al., 1979. Leg 64 seeks evidence on development of basins. Geotimes, 24:18-20.

Demars, K. R., and Nacci, V. A., 1978. Significance of Deep Sea Drilling Project sediment physical property data. Mar. Geotechnol., 3(2):151-170.
Hardin, B. O., and Richart, F. E., 1963. Elastic wave velocities in granular soils. J. Soil Mech. Found. Div., Am. Soc. Civ. Eng. 89: 33-65.

Lee, H. J., 1973. Measurements and estimates of engineering and other physical properties, Leg 19. In Creager, J. S., Scholl, D. W., et al., Init. Repts. DSDP, 19: Washington (U.S. Govt. Printing Office), 701-720.

Mayer, L. A., 1982. Physical properties of sediment recovered on Deep Sea Drilling Project Leg 68 with the hydraulic piston corer. In Prell, W. L., Gardner, J. V., et al., Init. Repts. DSDP, 68: Washington (U.S. Govt. Printing Office), 365-382.

Prell, W. L., Gardner, J. V., et al., 1982. Init. Repts. DSDP, 68: Washington (U.S. Govt. Printing Office).

Rocker, K., 1974. Physical properties measurements and test procedures for Leg 27. In Veevers, J. J., Heirtzler, J. R., et al., Init. Repts. DSDP, 27: Washington (U.S. Govt. Printing Office), 433-444.

Schrader, H.-J., Kelts, K., Curray, J,. et al., 1980. Laminated diatomaceous sediment from the Guaymas Basin slope (central Gulf of California): 250,000-year climate record. Science, 207:1207-1209.

Schultheiss, P. J., 1981. Simultaneous measurement of P and S wave velocities during conventional laboratory soil testing procedures. Mar. Geotechnol., 4(4):343-367.

1983. The influence of packing structure and effective stress on $\mathrm{V}_{\mathrm{s}}, \mathrm{V}_{\mathrm{p}}$ and the calculated dynamic and static moduli in sediments. Proc. Int. Conf. Acoustics and the Sea-bed, Bath, pp. 19-27.

Schultheiss, P. J., and Gunn, D. E., 1985. The consolidation and permeability of deep sea sediments. IOS report (in press).

Shirley, D. J., and Hampton, L. D., 1978. Shear wave measurements in laboratory sediments. J. Acoust. Soc. Am., 63:607-613.

Date of Initial Receipt: 3 November 1983

Date of Acceptance: 10 October 1984 


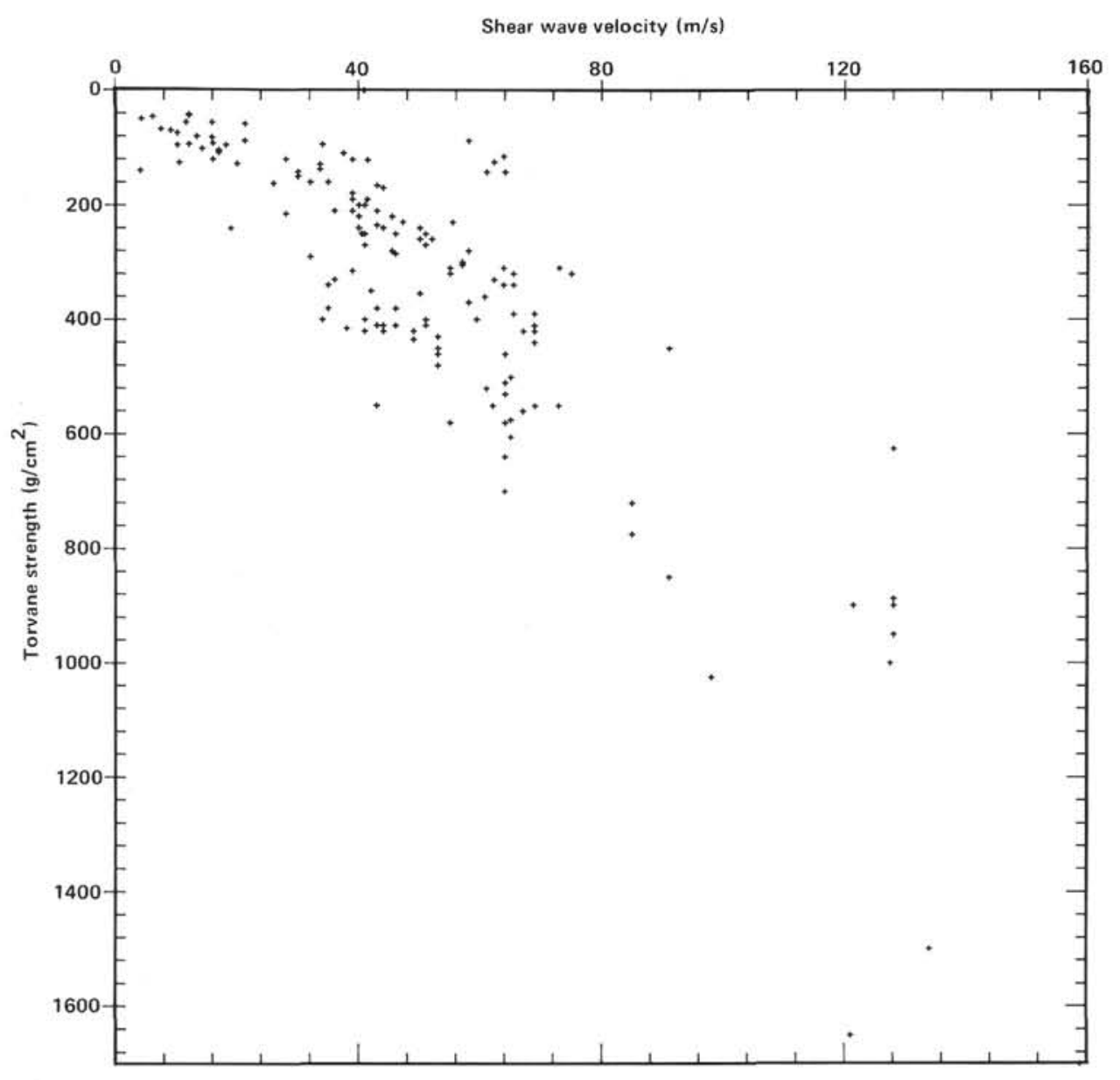

Figure 11. Torvane strength versus shear wave velocity for Sites 576, 577, 578, and 579. 

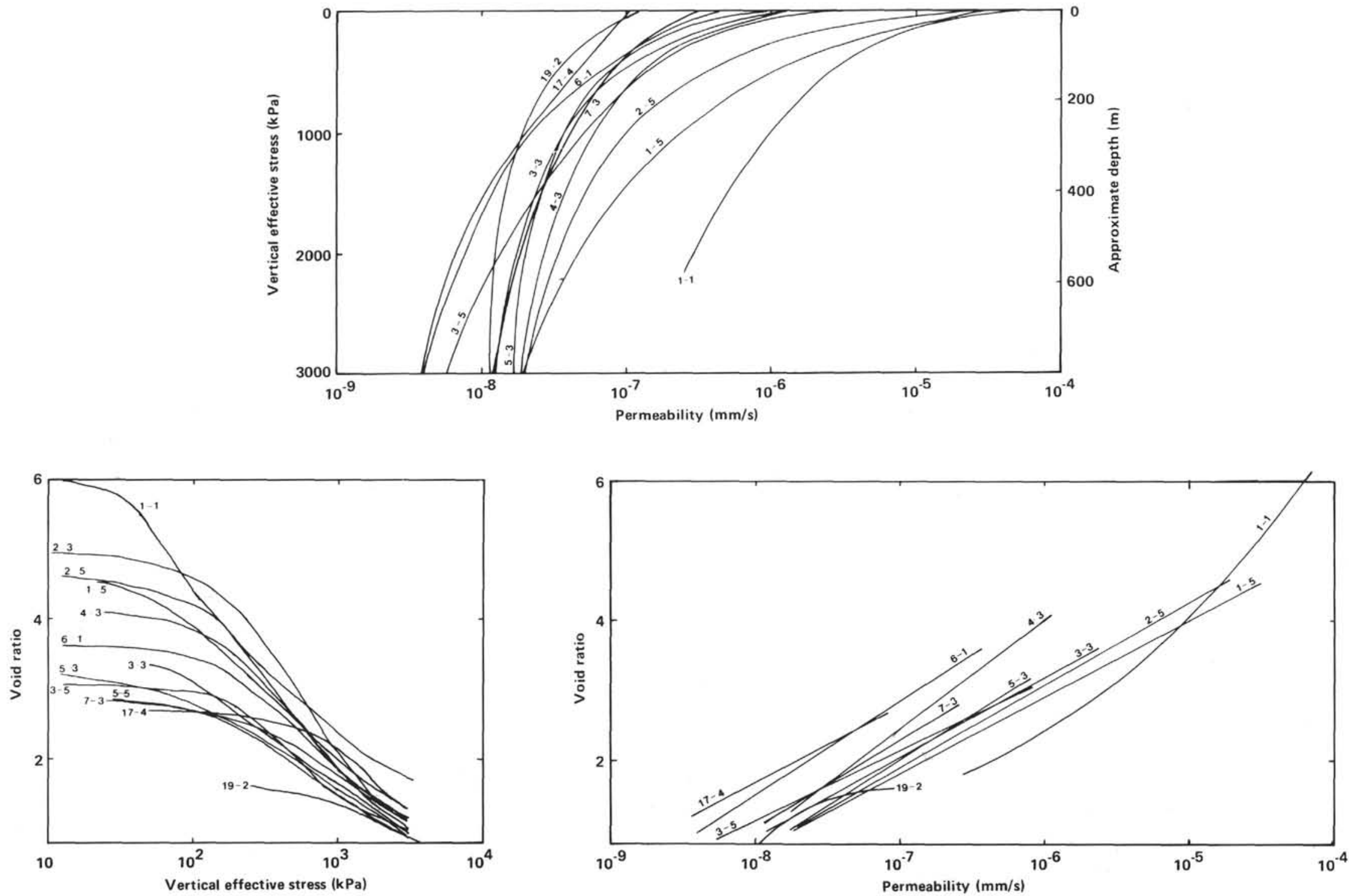

Figure 12. Consolidation and permeability data for red clay. Samples are from Holes 576A (Sections 1-1 to 7-3) and 578 (Sections 17-4 and 19-2). 

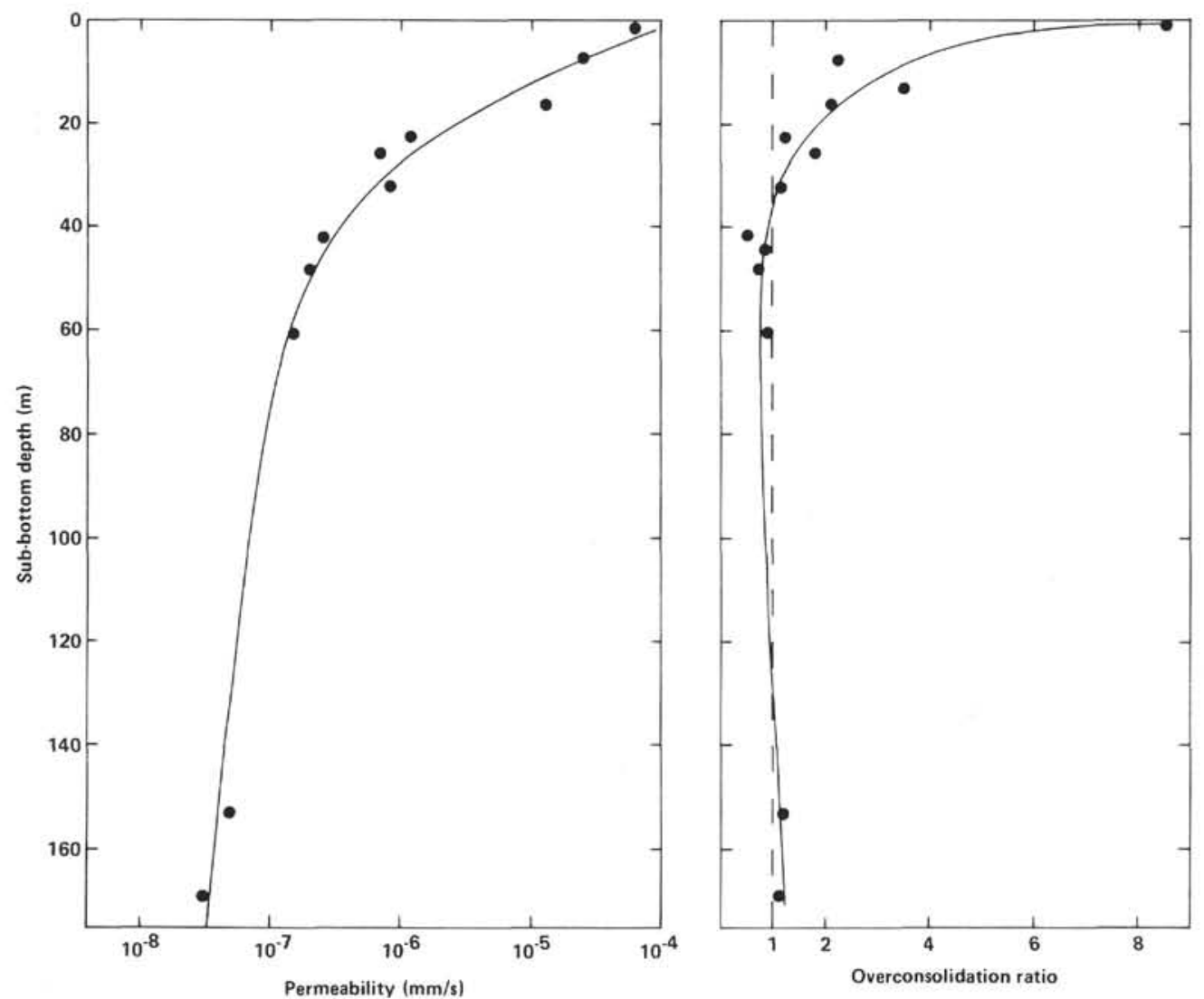

Figure 13. Permeability and overconsolidation ratio profiles in the red clay from Holes 576A and 578. 